\title{
Universal bounds on the electrical and elastic response of two-phase bodies and their application to bounding the volume fraction from boundary measurements
}

\author{
Graeme W. Milton \\ Department of Mathematics, University of Utah, Salt Lake City UT 84112, USA \\ email: milton@math.utah.edu, telephone: 1(801)581-6495, fax: 1(801)581-4148
}

\begin{abstract}
Universal bounds on the electrical and elastic response of two-phase (and multiphase) ellipsoidal or parallelopipedic bodies have been obtained by Nemat-Nasser and Hori. Here we show how their bounds can be improved and extended to bodies of arbitrary shape. Although our analysis is for two-phase bodies with isotropic phases it can easily be extended to multiphase bodies with anisotropic constituents. Our two-phase bounds can be used in an inverse fashion to bound the volume fractions occupied by the phases, and for electrical conductivity reduce to those of Capdeboscq and Vogelius when the volume fraction is asymptotically small. Other volume fraction bounds derived here utilize information obtained from thermal, magnetic, dielectric or elastic responses. One bound on the volume fraction can be obtained by simply immersing the body in a water filled cylinder with a piston at one end and measuring the change in water pressure when the piston is displaced by a known small amount. This bound may be particularly effective for estimating the volume of cavities in a body. We also obtain new bounds utilizing just one pair of (voltage, flux) electrical measurements at the boundary of the body.
\end{abstract}

Keywords: size estimation, universal bounds, volume fraction bounds.

\section{Introduction}

Berryman and Kohn (1990) found that classical variational principles could be used to obtain information about the conductivity inside a body from electrical measurements on the exterior. In this paper our main focus is on using classical variational principles and known bounds on the response of periodic composites to bound the volume fraction of one phase in a two-phase body $\Omega$ from measurements on the exterior of the body. Of course if one knows the mass densities of the two phases, the easiest way to do this is just to weigh the body. However this may not always be practical, or the densities of the two phases may be very close.

Two types of boundary conditions are most natural: what we call special Dirichlet conditions where affine Dirichlet conditions are imposed on the boundary of $\Omega$ (which 
would render the field inside $\Omega$ uniform if the body were homogeneous) or what we call special Neumann conditions where Neumann conditions are imposed that would render the field inside $\Omega$ uniform if the body were homogeneous. Bounds on the electrical and elastic response of the body to these special boundary conditions were obtained by Nemat-Nasser and Hori (1993, 1995), and were extended to piezoelectricity by Hori and Nemat-Nasser (1998). They called these bounds universal because they did not depend on any assumption about the microgeometry in the body. They obtained both elementary universal bounds based on the classical variational principles, and reviewed below in section 3, and universal bounds based on the Hashin-Shtrikman variational principles (Hashin and Shtrikman 1962, 1963). The latter bounds were obtained under the assumption that $\Omega$ is either an ellipsoid or a parallelopiped, but we will see here that they can easily be improved and generalized to bodies $\Omega$ of arbitrary shape. The key is to consider an assemblage of copies of $\Omega$ packed to fill all space, and then to use the bounds of Huet (1990) which relate the effective tensor of this composite to the responses of $\Omega$ under the special boundary conditions. Then existing bounds on the effective tensor [as surveyed in the books of Nemat-Nasser and Hori (1993), Cherkaev (2000), Allaire (2002), Milton (2002), and Tartar (2009)] can be directly applied to bound the responses of $\Omega$ under special boundary conditions (see sections 5,6,7, and 8). Since these bounds involve the volume fractions of the phases (and the moduli of the phases), they can be used in an inverse fashion to bound the volume fraction. As shown by Kang, Kim, and Milton (2011) the volume fraction bounds thus obtained for electrical conductivity generalize those obtained by Capdeboscq and Vogelius (2003, 2004) for the important case when the volume fraction is asymptotically small.

Given the close connection between bounds on effective tensors and bounds on the responses of $\Omega$ under special boundary condition, a natural question to ask is whether methods that have been used to derive bounds on effective tensors could be directly used to derive bounds on the response of $\Omega$ under more general boundary conditions. One such method is the Hashin-Strikman (1962, 1963) variational method and this lead Nemat-Nasser and Hori to their bounds for ellipsoidal or parallelopipedic $\Omega$. Another particularly successful method is the translation method (Tartar 1979; Lurie and Cherkaev 1982, 1984; Murat and Tartar 1985; Tartar 1985; Milton 1990) and indeed as shown in a companion paper (Kang, Kim, and Milton 2011) this method yields upper and lower bounds on the volume fraction in a two-phase body with general boundary conditions for two-dimensional conductivity without making any assumption on the shape of $\Omega$. For special boundary conditions the bounds thus derived reduce to the ones derived here.

We also provide (in section 4) some new conductivity bounds which just involve the results of just one (flux, voltage) pair measured at the boundary of $\Omega$, and which improve upon the elementary bounds of Nemat-Nasser and Hori (1993). Again these new bounds can be used in an inverse fashion to bound the volume fraction. Other volume fraction bounds using one measurement were derived by Kang, Seo, and Sheen (1997) Ikehata (1998), Alessandrini and Rosset (1998), Alessandrini, Rosset, and Seo (2000), and Alessandrini, Morassi, and Rosset (2002). These other bounds involve constants which are not easy to determine, making it difficult to make a general comparison with our new bounds.

The various bounds on the volume fraction we have derived are too numerous to summarize in this introduction. However we want to draw attention to the bounds (5.15) 
and (5.24) which are the natural extension of the famous Hashin and Shtrikman (1962) conductivity bounds to this problem. Also of particular note is the bound (8.26), which is one natural generalization of the bulk modulus bounds of Hashin and Shtrikman (1963) and Hill (1963), and implies that a bound on the volume fraction can be obtained by simply immersing the body in a water filled cylinder with a piston at one end and measuring the change in water pressure when the piston is displaced by a known small amount.

\section{The conductivity response tensors with special Dirich- let and special Neumann boundary conditions}

In electrical impedance tomography in a body $\Omega$ containing two isotropic components with (positive, scalar) conductivities $\sigma_{1}$ and $\sigma_{2}$ the potential $V$ satisfies

$$
\nabla \cdot \sigma \nabla V=0, \quad \text { where } \quad \sigma(\mathbf{x})=\chi(\mathbf{x}) \sigma_{1}+(1-\chi(\mathbf{x})) \sigma_{2},
$$

and $\chi(\mathbf{x})$ is the indicator function of component 1, taking the value 1 in component and 0 in component 2. Equivalently, in terms of the current field $\mathbf{j}(\mathbf{x})$ and electric field $\mathbf{e}(\mathbf{x})$ we have

$$
\nabla \cdot \mathbf{j}=0, \quad \mathbf{j}=\sigma \mathbf{e}, \quad \mathbf{e}=-\nabla V .
$$

Let us assume the components have been labeled so that $\sigma_{1} \geq \sigma_{2}$. We are given a set of Cauchy data, i.e. measurements of pairs $\left(V_{0}, q\right)$, where $V_{0}(\mathbf{x})$ and $q(\mathbf{x})$ are the boundary values of the voltage $V(\mathbf{x})$ and and flux $q(\mathbf{x})=-\mathbf{n} \cdot \mathbf{j}(\mathbf{x})$ at the boundary $\partial \Omega$ of $\Omega$, in which $\mathbf{n}(\mathbf{x})$ is the outwards normal to the boundary. From this boundary information we can immediately determine, using integration by parts, volume averages such as

$$
\begin{aligned}
\langle\mathbf{e} \cdot \mathbf{j}\rangle & =\frac{1}{|\Omega|} \int_{\partial \Omega}-V_{0}(\mathbf{j} \cdot \mathbf{n})=\frac{1}{|\Omega|} \int_{\partial \Omega} V_{0} q, \\
\langle\mathbf{e}\rangle & =\frac{1}{|\Omega|} \int_{\partial \Omega}-V_{0} \mathbf{n}, \\
\langle\mathbf{j}\rangle & =\frac{1}{|\Omega|} \int_{\partial \Omega}-\mathbf{x} q,
\end{aligned}
$$

where the angular brackets denote the volume average, i.e.

$$
\langle g\rangle=\frac{1}{|\Omega|} \int_{\Omega} g,
$$

for any quantity $g(\mathbf{x})$. From such averages our objective is to bound the volume fraction $f_{1}=\langle\chi\rangle$ of component 1 (and hence also the volume fraction $f_{2}=1-f_{1}$ of component 2).

To obtain good estimates of the volume fraction $f_{1}$ it makes physical sense to use measurements where the fields $\mathbf{e}(\mathbf{x})$ and $\mathbf{j}(\mathbf{x})$ probe well into the interior of $\Omega$. In this connection two sets of measurements are most natural. We could apply special Dirichlet boundary conditions

$$
V_{0}=-\mathbf{e}_{0} \cdot \mathbf{x}
$$


and measure $\mathbf{j}_{0}=\langle\mathbf{j}\rangle$. Here, according to (2.3), $\mathbf{e}_{0}$ equals $\langle\mathbf{e}\rangle$. Since $\mathbf{j}_{0}$ is linearly related to $\mathbf{e}_{0}$ we can write

$$
\mathbf{j}_{0}=\boldsymbol{\sigma}^{D} \mathbf{e}_{0},
$$

which defines the conductivity tensor $\boldsymbol{\sigma}^{D}$ ( $D$ for Dirichlet). To determine $\boldsymbol{\sigma}^{D}$ in dimension $d=2,3$ it of course suffices to measure $\mathbf{j}_{0}$ for $d$ linearly independent values of $\mathbf{e}_{0}$. Alternatively we could apply the special Neumann boundary conditions

$$
q=\mathbf{j}_{0} \cdot \mathbf{n},
$$

and measure $\mathbf{e}_{0}=\langle\mathbf{e}\rangle$. Again according to $(2.3), \mathbf{j}_{0}=\langle\mathbf{j}\rangle$. The linear relation between $\mathbf{e}_{0}$ and $\mathbf{j}_{0}$,

$$
\mathbf{e}_{0}=\left(\boldsymbol{\sigma}^{N}\right)^{-1} \mathbf{j}_{0}
$$

defines the resistivity tensor $\left(\boldsymbol{\sigma}^{N}\right)^{-1}$ and hence the conductivity tensor $\boldsymbol{\sigma}^{N}$ ( $N$ for Neumann): we will see later that $\left(\boldsymbol{\sigma}^{N}\right)^{-1}$ is invertible. To determine $\boldsymbol{\sigma}^{N}$ it suffices to measure $\mathbf{e}_{0}$ for $d$ linearly independent values of $\mathbf{j}_{0}$. With either of these two sorts of boundary conditions (but not in general) Hill (1963) has shown that

$$
\langle\mathbf{e} \cdot \mathbf{j}\rangle=\langle\mathbf{e}\rangle \cdot\langle\mathbf{j}\rangle
$$

as follows by substituting (2.5) or (2.7) in the first of equations (2.3). Using this relationship, and its obvious generalizations, it is easy to check that both $\boldsymbol{\sigma}^{D}$ and $\boldsymbol{\sigma}^{N}$ are self-adjoint. Thus if $\mathbf{e}^{\prime}(\mathbf{x})$ and $\mathbf{j}^{\prime}(\mathbf{x})$ denote the electric and current fields associated with the boundary conditions (2.5), with $\mathbf{e}_{0}$ replaced by $\mathbf{e}_{0}^{\prime}$, while keeping the same conductivity $\sigma(\mathbf{x})$ then

$$
\mathbf{e}_{0}^{\prime} \cdot \boldsymbol{\sigma}^{D} \mathbf{e}_{0}=\left\langle\mathbf{e}^{\prime} \cdot \mathbf{j}\right\rangle=\left\langle\mathbf{e}^{\prime} \sigma \mathbf{e}\right\rangle=\left\langle\mathbf{e} \cdot \mathbf{j}^{\prime}\right\rangle=\mathbf{e}_{0} \cdot \boldsymbol{\sigma}^{D} \mathbf{e}_{0}^{\prime},
$$

which implies $\boldsymbol{\sigma}^{D}$ is self-adjoint. By similar argument $\boldsymbol{\sigma}^{N}$ is self-adjoint.

\section{Known elementary bounds}

This section reviews the elementary bounds on $\boldsymbol{\sigma}^{D}$ and $\boldsymbol{\sigma}^{N}$ obtained by Nemat-Nasser and Hori (1993) and by Willis in a 1989 private communication to Nemat-Nasser and Hori. Their implications for bounding the volume fraction will be studied. We will make use of two classical variational principles: the Dirichlet variational principle that

$$
\begin{aligned}
& \underline{\mathbf{e}}(\mathbf{x})=-\nabla \underline{V}(\mathbf{x}) \quad \int_{\Omega} \underline{\mathbf{e}} \cdot \sigma \underline{\mathbf{e}}=\int_{\partial \Omega}-V_{0} q, \\
& \underline{V}(\mathbf{x})=V_{0}(\mathbf{x}) \text { on } \partial \Omega
\end{aligned}
$$

which is attained when $\underline{V}(\mathbf{x})=V(\mathbf{x})$, and the Neumann variational principle that

$$
\begin{aligned}
& \int_{\Omega} \underline{\mathbf{j}} \cdot \sigma^{-1} \underline{\mathbf{j}}=\int_{\partial \Omega} V_{0} q, \\
& \nabla \cdot \overline{\mathbf{j}}(\mathbf{x})=0 \\
& \mathbf{n} \cdot \underline{\mathbf{j}}(\mathbf{x})=-q(\mathbf{x}) \text { on } \partial \Omega
\end{aligned}
$$


which is attained when $j(\mathbf{x})=\mathbf{j}(\mathbf{x})$. With the special Dirichlet boundary conditions (2.5) the Dirichlet variational principle implies

$$
\begin{aligned}
& \underline{\mathbf{e}}(\mathbf{x})=-\nabla \underline{V}(\mathbf{x}) \quad\langle\underline{\mathbf{e}} \cdot \sigma \underline{\mathbf{e}}\rangle=\mathbf{e}_{0} \cdot \boldsymbol{\sigma}^{D} \mathbf{e}_{0} . \\
& \underline{V}(\mathbf{x})=-\mathbf{e}_{0} \cdot \mathbf{x} \text { on } \partial \Omega
\end{aligned}
$$

Taking a trial potential $\underline{V}=-\mathbf{e}_{0} \cdot \mathbf{x}$ produces the elementary upper bound on $\mathbf{e}_{0} \cdot \boldsymbol{\sigma}^{D} \mathbf{e}_{0}$

$$
\mathbf{e}_{0} \cdot \boldsymbol{\sigma}^{D} \mathbf{e}_{0} \leq\langle\sigma\rangle \mathbf{e}_{0} \cdot \mathbf{e}_{0},
$$

given by Nemat-Nasser and Hori (1993). To obtain a lower bound observe, following a standard argument, that the left hand side of (3.3) is surely decreased if we take the minimum over a larger class of trial fields. Since the constraints on $\underline{\mathbf{e}}(\mathbf{x})$ imply $\langle\underline{\mathbf{e}}\rangle=\mathbf{e}_{0}$ let us replace them by this weaker constraint to obtain the inequality

$$
\begin{gathered}
\mathbf{e}_{0} \cdot \boldsymbol{\sigma}^{D} \mathbf{e}_{0} \geq \min _{\underline{\mathbf{e}}(\mathbf{x})}\langle\underline{\mathbf{e}} \cdot \sigma \underline{\mathbf{e}}\rangle, \\
\langle\underline{\mathbf{e}}\rangle=\mathbf{e}_{0}
\end{gathered}
$$

where the minimum is now over fields $\underline{\mathbf{e}}$ which are not necessarily curl-free. Using Lagrange multipliers one finds that the minimum is attained when $\underline{\mathbf{e}}=\sigma^{-1}\left\langle\sigma^{-1}\right\rangle^{-1} \mathbf{e}_{0}$ and so we obtain the lower bound

$$
\mathbf{e}_{0} \cdot \boldsymbol{\sigma}^{D} \mathbf{e}_{0} \geq\left\langle\sigma^{-1}\right\rangle^{-1} \mathbf{e}_{0} \cdot \mathbf{e}_{0}
$$

of Nemat-Nasser and Hori (1993). Taken together, (3.4) and (3.6) imply the lower and upper bounds

$$
\left(\frac{\mathbf{e}_{0} \cdot \boldsymbol{\sigma}^{D} \mathbf{e}_{0}}{\mathbf{e}_{0} \cdot \mathbf{e}_{0}}-\sigma_{2}\right) /\left(\sigma_{1}-\sigma_{2}\right) \leq f_{1} \leq\left(\sigma_{2}^{-1}-\frac{\mathbf{e}_{0} \cdot \mathbf{e}_{0}}{\mathbf{e}_{0} \cdot \boldsymbol{\sigma}^{D} \mathbf{e}_{0}}\right) /\left(\sigma_{2}^{-1}-\sigma_{1}^{-1}\right)
$$

on the volume fraction $f_{1}$. These bounds give useful information even if we only know $\mathbf{j}_{0}=\boldsymbol{\sigma}^{D} \mathbf{e}_{0}$ for only one value of $\mathbf{e}_{0}$, i.e. if we only take one measurement. These bounds (3.7) are sharp in the sense that the lower bound is approached artitrarily closely if $\Omega$ is filled with a periodic laminate of components 1 and 2, oriented with the normal to the layers orthogonal to $\mathbf{e}_{0}$ and we let the period length go to zero, while the upper bound is approached artitrarily closely for the same geometry, but oriented with the normal to the layers parallel to $\mathbf{e}_{0}$. If the full tensor $\boldsymbol{\sigma}^{D}$ is known, from $d=2,3$ measurements of pairs $\left(\mathbf{e}_{0}, \mathbf{j}_{0}\right)$ then we can take the intersection of the bounds (3.7) as $\mathbf{e}_{0}$ is varied, and so obtain

$$
\left(\lambda_{+}^{D}-\sigma_{2}\right) /\left(\sigma_{1}-\sigma_{2}\right) \leq f_{1} \leq\left(1 / \sigma_{2}-1 / \lambda_{-}^{D}\right) /\left(\sigma_{2}^{-1}-\sigma_{1}^{-1}\right),
$$

where $\lambda_{+}^{D}$ and $\lambda_{-}^{D}$ are the maximum and minimum eigenvalues of $\boldsymbol{\sigma}^{D}$. However we will see in the next section that an additional and typically sharper upper bound on $f_{1}$ can be obtained.

With the special Neumann boundary conditions (2.7) the variational principle (3.2) implies

$$
\begin{aligned}
& \min _{\mathbf{j}(\mathbf{x})} \quad\left\langle\underline{\mathbf{j}} \cdot \sigma^{-1} \underline{\mathbf{j}}\right\rangle=\mathbf{j}_{0} \cdot\left(\boldsymbol{\sigma}^{N}\right)^{-1} \mathbf{j}_{0} . \\
& \nabla \cdot \underline{\mathbf{j}}(\mathbf{x})=0 \\
& \mathbf{n} \cdot \underline{\mathbf{j}}(\mathbf{x})=\mathbf{n} \cdot \mathbf{j}_{0} \text { on } \partial \Omega
\end{aligned}
$$


By taking a constant trial field $\underline{\mathbf{j}}(\mathbf{x})=\mathbf{j}_{0}$ or alternatively by taking the minimum over the larger class of trial fields satisfying only $\langle\underline{\mathbf{j}}\rangle=\mathbf{j}_{0}$ we obtain the bounds

$$
\langle\sigma\rangle^{-1} \mathbf{j}_{0} \cdot \mathbf{j}_{0} \leq \mathbf{j}_{0} \cdot\left(\boldsymbol{\sigma}^{N}\right)^{-1} \mathbf{j}_{0} \leq\left\langle\sigma^{-1}\right\rangle \mathbf{j}_{0} \cdot \mathbf{j}_{0}
$$

of Nemat-Nasser and Hori (1993) which imply

$$
\left(\frac{\mathbf{j}_{0} \cdot \mathbf{j}_{0}}{\mathbf{j}_{0} \cdot\left(\boldsymbol{\sigma}^{N}\right)^{-1} \mathbf{j}_{0}}-\sigma_{2}\right) /\left(\sigma_{1}-\sigma_{2}\right) \leq f_{1} \leq\left(\sigma_{2}^{-1}-\frac{\mathbf{j}_{0} \cdot\left(\boldsymbol{\sigma}^{N}\right)^{-1} \mathbf{j}_{0}}{\mathbf{j}_{0} \cdot \mathbf{j}_{0}}\right) /\left(\sigma_{2}^{-1}-\sigma_{1}^{-1}\right)
$$

These bounds are applicable even if we only know $\mathbf{e}_{0}=\left(\boldsymbol{\sigma}^{N}\right)^{-1} \mathbf{j}_{0}$ for only one value of $\mathbf{j}_{0}$. For comparison, with these special Neumann boundary conditions (2.7), the bounds in Theorem 3.1 of Kang, Seo, and Sheen (1997) coupled with the improvement in proposition 0 of Ikehata (1998), with $\sigma_{1}=k>1, \sigma_{2}=1$ and $\mathbf{j}_{0} \cdot \mathbf{j}_{0}=1$, imply

$$
\frac{1}{k-1}\left(1-\mathbf{j}_{0} \cdot\left(\boldsymbol{\sigma}^{N}\right)^{-1} \mathbf{j}_{0}\right) \leq f_{1} \leq \frac{k}{k-1}\left(1-\mathbf{j}_{0} \cdot\left(\boldsymbol{\sigma}^{N}\right)^{-1} \mathbf{j}_{0}\right)
$$

In this case it is easy to check that the upper bounds in (3.11) and (3.12) coincide while the lower bound in (3.11) is tighter. The bounds (3.11) are each approached arbitrarily closely if $\Omega$ is filled with a periodic laminate of components 1 and 2 , oriented with $\mathbf{j}_{0}$ either parallel or orthogonal to the layers and we let the period length go to zero.

In summary, (3.4), (3.6) and (3.10) imply the matrix inequalities

$$
\left\langle\sigma^{-1}\right\rangle^{-1} \mathbf{I} \leq \boldsymbol{\sigma}^{D} \leq\langle\sigma\rangle \mathbf{I}, \quad\left\langle\sigma^{-1}\right\rangle^{-1} \mathbf{I} \leq \boldsymbol{\sigma}^{N} \leq\langle\sigma\rangle \mathbf{I}
$$

of Nemat-Nasser and Hori (1993).

For artitrary boundary conditions, i.e. for any $\mathbf{e}$ and $\mathbf{j}$ satisfying (2.2) within $\Omega$, we have the bounds

$$
\langle\mathbf{e} \cdot \mathbf{j}\rangle \geq \mathbf{e}_{0} \cdot \boldsymbol{\sigma}^{N} \mathbf{e}_{0}, \quad\langle\mathbf{e} \cdot \mathbf{j}\rangle \geq \mathbf{j}_{0}\left(\boldsymbol{\sigma}^{D}\right)^{-1} \mathbf{j}_{0}
$$

where $\mathbf{e}_{0}=\langle\mathbf{e}\rangle$ and $\mathbf{j}_{0}=\langle\mathbf{j}\rangle$, due to Willis in a 1989 private communication to NematNasser and Hori, and presented by Nemat-Nasser and Hori (1993). In conjunction with (3.13) they imply the volume fraction bounds,

$$
\left(\frac{\mathbf{j}_{0} \cdot \mathbf{j}_{0}}{\langle\mathbf{e} \cdot \mathbf{j}\rangle}-\sigma_{2}\right) /\left(\sigma_{1}-\sigma_{2}\right) \leq f_{1} \leq\left(\sigma_{2}^{-1}-\frac{\mathbf{e}_{0} \cdot \mathbf{e}_{0}}{\langle\mathbf{e} \cdot \mathbf{j}\rangle}\right) /\left(\sigma_{2}^{-1}-\sigma_{1}^{-1}\right) .
$$

\section{New bounds with one measurement}

If we have measurements of $\langle\mathbf{e} \cdot \mathbf{j}\rangle$ and both vectors $\mathbf{e}_{0}$ and $\mathbf{j}_{0}$ for arbitrary boundary conditions then the bounds (3.14) and (3.15) can be improved. The classical variational principle (3.1) implies

$$
\begin{aligned}
& \min _{\underline{\mathbf{e}}(\mathbf{x})=-\nabla \underline{V}(\mathbf{x})}\langle\underline{\mathbf{e}} \cdot \sigma \underline{\mathbf{e}}\rangle,=\langle\mathbf{e} \cdot \mathbf{j}\rangle \\
& \underline{V}(\mathbf{x})=V_{0}(\mathbf{x}) \text { on } \partial \Omega \\
& \langle\sigma \underline{\mathbf{e}}\rangle=\mathbf{j}_{0}
\end{aligned}
$$


where we have chosen to add the constraint that $\langle\sigma \underline{\mathbf{e}}\rangle=\mathbf{j}_{0}$ since we know that without this constraint the minimizer $\underline{\mathbf{e}}=\mathbf{e}$ satisfies $\langle\sigma \mathbf{e}\rangle=\mathbf{j}_{0}$. We surely obtain something lower if take the minimum over the larger class of fields satisfying only $\langle\underline{\mathbf{e}}\rangle=\mathbf{e}_{0}$ and $\langle\sigma \underline{\mathbf{e}}\rangle=\mathbf{j}_{0}$. Thus we obtain the inequality

$$
\begin{aligned}
& \min _{\underline{\mathbf{e}}(\mathbf{x})}\langle\underline{\mathbf{e}} \cdot \sigma \underline{\mathbf{e}}\rangle \leq\langle\mathbf{e} \cdot \mathbf{j}\rangle . \\
& \langle\underline{\mathbf{e}}\rangle=\mathbf{e}_{0} \\
& \langle\sigma \underline{\mathbf{e}}\rangle=\mathbf{j}_{0}
\end{aligned}
$$

By introducing two vector valued Lagrange multipliers associated with the two vector valued constraints we find that the minimum is attained when

$$
\underline{\mathbf{e}}(\mathbf{x})=\mathbf{e}_{0}+\left(\left\langle\sigma^{-1}\right\rangle-\sigma^{-1}(\mathbf{x})\right)\left(\langle\sigma\rangle\left\langle\sigma^{-1}\right\rangle-1\right)^{-1}\left(\mathbf{j}_{0}-\langle\sigma\rangle \mathbf{e}_{0}\right) .
$$

Substituting this back in (4.2) gives the bound

$$
\left(\langle\sigma\rangle\left\langle\sigma^{-1}\right\rangle-1\right)\left(\langle\mathbf{e} \cdot \mathbf{j}\rangle-\mathbf{e}_{0} \cdot \mathbf{j}_{0}\right) \geq\left(\mathbf{j}_{0}-\langle\sigma\rangle \mathbf{e}_{0}\right) \cdot\left(\left\langle\sigma^{-1}\right\rangle \mathbf{j}_{0}-\mathbf{e}_{0}\right) .
$$

If, with general boundary conditions, we are interested in bounding the volume fraction $f_{1}$ given measured values of $\langle\mathbf{e} \cdot \mathbf{j}\rangle, \mathbf{e}_{0}$ and $\mathbf{j}_{0}$ then the difference between the left hand side and right hand side of (4.4) is a quadratic in $f_{1}$ whose two roots give upper and lower bounds on $f_{1}$. (Unless the roots happen to be complex, in which case there is no configuration of the two phases within $\Omega$ which produce the measured $\langle\mathbf{e} \cdot \mathbf{j}\rangle, \mathbf{e}_{0}$ and $\mathbf{j}_{0}$, indicating the presence of other phases or indicating an error in measurements.)

In the particular cases of either special Dirichlet or special Neumann boundary conditions, (2.5) or (2.7), the left hand side of (4.4) vanishes (see (2.9)) and we obtain the reduced bounds

$$
0 \geq\left(\mathbf{j}_{0}-\langle\sigma\rangle \mathbf{e}_{0}\right) \cdot\left(\left\langle\sigma^{-1}\right\rangle \mathbf{j}_{0}-\mathbf{e}_{0}\right),
$$

which are in fact implied by the matrix inequalities (3.13). This bound (4.5) is optimal. For any given fixed $\mathbf{e}_{0}$, and fixed volume fraction $f_{1}$, the vector $\mathbf{j}_{0}$ has an endpoint which is constrained by (4.5) to lie within a sphere (disk in two dimensions) centered at $\left(\langle\sigma\rangle+\left\langle\sigma^{-1}\right\rangle^{-1}\right) \mathbf{e}_{0} / 2$. When $\Omega$ is filled with a periodic laminate of the two phases with interfaces orthogonal to some unit vector $\mathbf{m}$, and we let the period length go to zero, then the endpoint of the vector $\mathbf{j}_{0}$ covers the entire surface of this sphere (disk) as $\mathbf{m}$ ranges over all unit vectors. These bounds are the analogs, for arbitrary bodies $\Omega$, of bounds on possible $\left(\mathbf{e}_{0}, \mathbf{j}_{0}\right)$ pairs for composites derived by Raŭtum (1983) and Tartar (1995). If we are given $\mathbf{e}_{0}$ and $\mathbf{j}_{0}$ and want to bound $f_{1}$ then we should find the range of $f_{1}$ where the sphere (or disk) contains the endpoint of the vector $\mathbf{j}_{0}$. The endpoints of this range are the roots of the right hand side of (4.5) which is a quadratic function of $f_{1}$.

Knowledge of $\mathbf{e}_{0}$ and $\mathbf{j}_{0}$ is equivalent to knowledge of $\langle\mathbf{e} \cdot \mathbf{v}\rangle$ and $\langle\mathbf{v} \cdot \mathbf{j}\rangle$ for all constant fields $\mathbf{v}$. A more general alternative is to use the information about

$$
\begin{array}{r}
a_{i}=\left\langle\mathbf{e} \cdot \mathbf{j}_{i}\right\rangle=\frac{1}{|\Omega|} \int_{\partial \Omega}-V_{0}\left(\mathbf{j}_{i} \cdot \mathbf{n}\right), \\
b_{k}=\left\langle\nabla V_{k} \cdot \mathbf{j}\right\rangle=\frac{1}{|\Omega|} \int_{\partial \Omega}-V_{k}(\mathbf{j} \cdot \mathbf{n}),
\end{array}
$$


for a given set of "comparison flux fields" $\mathbf{j}_{i}(\mathbf{x})$ satisfying $\nabla \cdot \mathbf{j}_{i}=0, i=1,2, \ldots n$ and "comparison potentials" $V_{k}(\mathbf{x}), k=1,2, \ldots m$. Suppose, for example, that we have just one comparison flux field $\mathbf{j}_{1}$. We have the variational principle

$$
\begin{aligned}
& \underline{\mathbf{e}}(\mathbf{x})=-\nabla \underline{V}(\mathbf{x}) \quad\langle\underline{\mathbf{e}} \cdot \sigma \underline{\mathbf{e}}\rangle=\langle\mathbf{e} \cdot \mathbf{j}\rangle, \\
& \underline{V}(\mathbf{x})=V_{0}(\mathbf{x}) \text { on } \partial \Omega \\
& \left\langle\underline{\mathbf{e}} \cdot \mathbf{j}_{1}\right\rangle=a_{1}
\end{aligned}
$$

where we have chosen to add the constraint that $\left\langle\underline{\mathbf{e}} \cdot \mathbf{j}_{1}\right\rangle=a_{1}$ since we know that without this constraint the minimizer $\underline{\mathbf{e}}=\mathbf{e}$ satisfies $\left\langle\mathbf{e} \cdot \mathbf{j}_{1}\right\rangle=a_{1}$. This implies the inequality

$$
\begin{aligned}
& \langle\mathbf{e} \cdot \mathbf{j}\rangle \geq \min _{\underline{\mathbf{e}}(\mathbf{x})}\langle\underline{\mathbf{e}} \cdot \sigma \underline{\mathbf{e}}\rangle . \\
& \left\langle\underline{\mathbf{e}} \cdot \mathbf{j}_{1}\right\rangle=a_{1}
\end{aligned}
$$

By introducing a Lagrange multiplier associated with the constraint $\left\langle\mathbf{e} \cdot \mathbf{j}_{1}\right\rangle=a_{1}$ we see the minimum occurs when

$$
\underline{\mathbf{e}}=a_{1} \sigma^{-1} \mathbf{j}_{1} /\left\langle\mathbf{j}_{1} \cdot \sigma^{-1} \mathbf{j}_{1}\right\rangle
$$

giving the inequality

$$
\langle\mathbf{e} \cdot \mathbf{j}\rangle \geq a_{1}^{2} /\left\langle\mathbf{j}_{1} \cdot \sigma^{-1} \mathbf{j}_{1}\right\rangle .
$$

This inequality gives information about $\sigma(\mathbf{x})$ through $\left\langle\mathbf{j}_{1} \cdot \sigma^{-1} \mathbf{j}_{1}\right\rangle$. If we only want bounds which involve the volume fraction we should choose $\mathbf{j}_{1}(\mathbf{x})$ with

$$
\left|\mathbf{j}_{1}(\mathbf{x})\right|=1 \quad \text { for all } \mathbf{x} \in \Omega \text {. }
$$

There are many divergence free fields $\mathbf{j}_{1}(\mathbf{x})$ which satisfy this constraint. For example in two dimensions we can take

$$
\mathbf{j}_{1}=\left(\partial \phi / \partial x_{2},-\partial \phi / \partial x_{1}\right), \text { with }|\nabla \phi(\mathbf{x})|=1 \quad \text { for all } \mathbf{x} \in \Omega \text {. }
$$

Thus $\phi(\mathbf{x})$ satisfies an Eikonal equation, and we could take $\phi(\mathbf{x})$ to be the shortest distance between $\mathbf{x}$ and a curve outside $\Omega$. Once (4.11) is satisfied (4.10) implies the volume fraction bound

$$
f_{1} \leq\left(\sigma_{2}^{-1}-\frac{a_{1}^{2}}{\langle\mathbf{e} \cdot \mathbf{j}\rangle}\right) /\left(\sigma_{2}^{-1}-\sigma_{1}^{-1}\right) .
$$

In the special case when $\mathbf{j}_{1}=\mathbf{e}_{0} /\left|\mathbf{e}_{0}\right|$ this reduces to the upper bound on $f_{1}$ given by (3.15).

An important question is whether this new bound is sharp, and if so for what $\sigma(\mathbf{x})$ ? The new bound will be sharp when $\mathbf{e}=\underline{\mathbf{e}}$ where $\underline{\mathbf{e}}$ is given by (4.9). In that case

$$
\mathbf{j}(\mathbf{x})=a_{1} \mathbf{j}_{1} /\left\langle\mathbf{j}_{1} \cdot \sigma^{-1} \mathbf{j}_{1}\right\rangle
$$

has zero divergence because it is proportional to $\mathbf{j}_{1}$. Let us impose the Neumann boundary condition

$$
\mathbf{j}(\mathbf{x}) \cdot \mathbf{n}=\mathbf{j}_{1} \cdot \mathbf{n} \quad \text { for all } \mathbf{x} \in \partial \Omega
$$




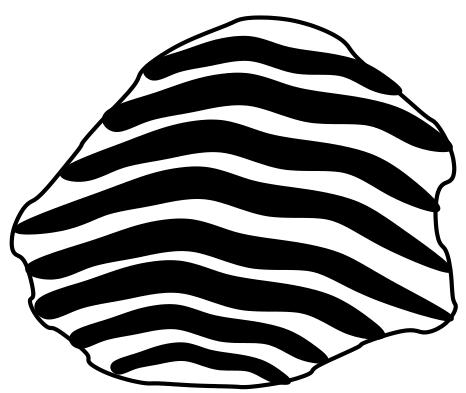

Figure 1: A schematic of the type of layered microstructure achieving the volume fraction bound (4.13), where the black regions denote one phase, and the white regions the other phase. The layer widths should be much finer than the size of $\Omega$.

and look for a $\sigma(\mathbf{x})$ so $\mathbf{j}(\mathbf{x})=\mathbf{j}_{1}(\mathbf{x})$ and $\mathbf{e}(\mathbf{x})=\sigma^{-1} \mathbf{j}_{1}(\mathbf{x})$ is curl-free. Now as schematically represented by figure 1, choose $\sigma(\mathbf{x})$ to correspond to a finely layered composite with layers orthogonal to the streamlines of $\mathbf{j}_{1}(\mathbf{x})$, and with phase 1 occupying a local volume fraction $p(\mathbf{x})$. This composite will support a current field $\mathbf{j}(\mathbf{x})=\mathbf{j}_{1}(\mathbf{x})$ and an electric field $\mathbf{e}(\mathbf{x})=\sigma^{-1} \mathbf{j}_{1}(\mathbf{x})$ provided

$$
\nabla \times \mathbf{e}_{0}=0, \quad \mathbf{e}_{0} \equiv\left[\sigma_{2}^{-1}-p(\mathbf{x})\left(\sigma_{2}^{-1}-\sigma_{1}^{-1}\right)\right] \mathbf{j}_{1}(\mathbf{x}) .
$$

Here $\mathbf{e}_{0}(\mathbf{x})$ is the weak limit (local volume average) of $\mathbf{e}(\mathbf{x})$ as the layer spacing goes to zero. In two dimensions, given $\mathbf{j}_{1}(\mathbf{x})$ we could look for solutions for $p(\mathbf{x})$ such that (4.16) is satisfied and $0 \leq p(\mathbf{x}) \leq 1$ in $\Omega$. We expect such solutions to exist for a wide class of fields $\mathbf{j}_{1}(\mathbf{x})$. This example shows that non-constant "comparison flux fields" can lead to sharp bounds on the volume fraction. In three dimensions we only expect to find a solution of the vector equation (4.16) for the scalar field $p(\mathbf{x})$ if $\mathbf{j}_{1}(\mathbf{x})$ satisfies some additional conditions.

\section{Relationship to bounding effective tensors of com- posites}

Consider a periodic composite obtained by taking the unit cell boundaries outside $\Omega \equiv \Omega_{1}$ and almost filling the rest of the unit cell by non-intersecting rescaled and translated copies $\Omega_{i}, i=2, \ldots, n$ of $\Omega$, as illustrated in figure 2. The remainder of the unit cell is filled by phase 2 with conductivity $\sigma_{2}$. The unit cell structure is periodically repeated to fill all space. Let $\sigma_{C}(\mathbf{x})$ ( $C$ for composite) denote this effective conductivity, i.e. in the 


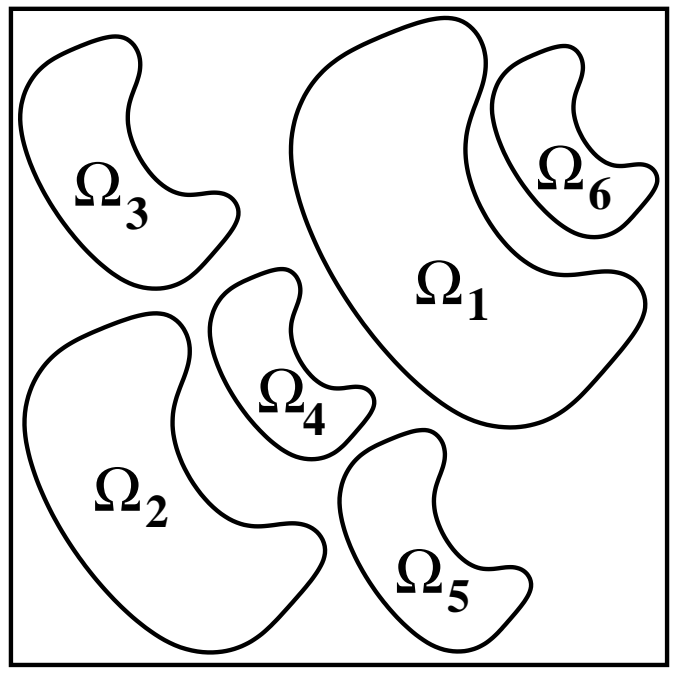

Figure 2: A period cell containing rescaled copies of $\Omega$.

unit cell

$$
\begin{aligned}
\sigma_{C}(\mathbf{x}) & =\sigma\left(\mathbf{x} / a_{i}+\mathbf{b}_{i}\right) \text { in } \Omega_{i}, \quad i=1,2, \ldots, N \\
& =\sigma_{2} \text { elsewhere outside } \cup_{i} \Omega_{i},
\end{aligned}
$$

where the scaling constants $a_{i}$ and translation vectors $\mathbf{b}_{i}$ (with $a_{1}=1$ and $\mathbf{b}_{1}=0$ ) are determined by the size and position of each copy $\Omega_{i}$, so that $\mathbf{x} / a_{i}+\mathbf{b}_{i}$ is on the boundary of $\Omega$ if and only if $\mathbf{x}$ is on the boundary of $\Omega_{i}$. Let $p_{n}$ denote the volume fraction in the unit cell occupied by the material with conductivity $\sigma_{2}$. Let $\boldsymbol{\sigma}_{n}^{*}$ denote the (matrix valued) effective conductivity of this composite, which in general depends upon the relative positions of the copies $\Omega_{i}$ within the unit cell.

We have the classical variational inequality

$$
\mathbf{e}_{0} \cdot \boldsymbol{\sigma}_{n}^{*} \mathbf{e}_{0} \leq\left\langle\underline{\mathbf{e}}_{C} \cdot \sigma_{C} \underline{\mathbf{e}}_{C}\right\rangle
$$

which holds for any trial electric field $\underline{\mathbf{e}}_{C}$ satisfying

$$
\nabla \times \underline{\mathbf{e}}_{C}=0, \quad \underline{\mathbf{e}}_{C} \text { periodic, } \quad\left\langle\underline{\mathbf{e}}_{C}\right\rangle=\mathbf{e}_{0},
$$

where now the volume averages are over the entire unit cell, rather than just $\Omega$. In particular, letting $\mathbf{e}(\mathbf{x})$ denote the electric field within $\Omega$ when the special Dirichlet boundary conditions (2.5) are applied, we may take in the unit cell

$$
\begin{aligned}
\underline{\mathbf{e}}_{C}(\mathbf{x}) & =\mathbf{e}\left(\mathbf{x} / a_{i}+\mathbf{b}_{i}\right) \text { in } \Omega_{i}, \quad i=1,2, \ldots, N \\
& =\mathbf{e}_{0} \text { elsewhere outside } \cup_{i} \Omega_{i},
\end{aligned}
$$

and periodically extend it. Then we get

$$
\left\langle\underline{\mathbf{e}}_{C} \cdot \sigma_{C} \underline{\mathbf{e}}_{C}\right\rangle=p_{n} \sigma_{2} \mathbf{e}_{0} \cdot \mathbf{e}_{0}
$$




$$
\begin{aligned}
& +\left(1-p_{n}\right) \sum_{i=1}^{N}\left\langle\mathbf{e}\left(\mathbf{x} / a_{i}+\mathbf{b}_{i}\right) \cdot \sigma\left(\mathbf{x} / a_{i}+\mathbf{b}_{i}\right) \mathbf{e}\left(\mathbf{x} / a_{i}+\mathbf{b}_{i}\right)\right\rangle_{\Omega_{i}} \\
= & p_{n} \sigma_{2} \mathbf{e}_{0} \cdot \mathbf{e}_{0}+\left(1-p_{n}\right)\langle\mathbf{e}(\mathbf{x}) \cdot \sigma(\mathbf{x}) \mathbf{e}(\mathbf{x})\rangle_{\Omega} \\
= & p_{n} \sigma_{2} \mathbf{e}_{0} \cdot \mathbf{e}_{0}+\left(1-p_{n}\right) \mathbf{e}_{0} \cdot \boldsymbol{\sigma}^{D} \mathbf{e}_{0},
\end{aligned}
$$

where $\langle\cdot\rangle_{\Omega_{i}}$ denotes an average over the region $\Omega_{i}$. Combined with the variational inequality (5.2) this implies the bound

$$
\mathbf{e}_{0} \cdot \boldsymbol{\sigma}_{n}^{*} \mathbf{e}_{0} \leq p_{n} \sigma_{2} \mathbf{e}_{0} \cdot \mathbf{e}_{0}+\left(1-p_{n}\right) \mathbf{e}_{0} \cdot \boldsymbol{\sigma}^{D} \mathbf{e}_{0} \leq \mathbf{e}_{0} \cdot \boldsymbol{\sigma}^{D} \mathbf{e}_{0},
$$

where we have used the inequality $\boldsymbol{\sigma}^{D} \geq \sigma_{2} \mathbf{I}$ implied by (3.13). Thus we get

$$
\boldsymbol{\sigma}_{n}^{*} \leq \boldsymbol{\sigma}^{D} .
$$

This composite has a volume fraction $f_{1}^{\prime}=\left(1-p_{n}\right) f_{1}$ of phase 1 . Thus any bound "from below" on the effective conductivity $\boldsymbol{\sigma}_{n}^{*}$, applicable to composites having a volume fraction $f_{1}^{\prime}$ of phase 1 , immediately translates into bound "from below" on $\boldsymbol{\sigma}^{D}$. Now consider what happens as we increase $N$, inserting more and more regions $\Omega_{i}$, while leaving undisturbed the regions $\Omega_{i}$ already in place, so that $p_{n} \rightarrow 0$ as $n \rightarrow \infty$. We are assured that this is possible. Rescaled copies of any shaped region can be packed to fill all space: see, for example, Theorem A.1 in Benveniste and Milton (2003). Define

$$
\boldsymbol{\sigma}^{*}=\lim _{n \rightarrow \infty} \boldsymbol{\sigma}_{n}^{*}
$$

We are assured this limit exists since if we change the geometry in some small volume then the effective conductivity (assuming $\sigma_{1}$ and $\sigma_{2}$ are strictly positive and finite) is perturbed only by a small amount (Zhikov, Kozlov, and Oleinik 1994). We will call $\boldsymbol{\sigma}^{*}$ the effective conductivity tensor of an assemblage of rescaled copies of $\Omega$ packed to fill all space. Then (5.7) implies

$$
\boldsymbol{\sigma}^{*} \leq \boldsymbol{\sigma}^{D}
$$

which is essentially the bound of Huet (1990) applied to this assemblage. Assume the bound is continuous with respect to $f_{1}^{\prime}$ at the point $f_{1}^{\prime}=f_{1}$, as expected. Then taking the limit $n \rightarrow \infty$ the "lower bound" on the effective tensor of composites having volume fraction $f_{1}$ must also be a lower bound on $\boldsymbol{\sigma}^{D}$.

In particular, the harmonic mean bound $\boldsymbol{\sigma}^{*} \geq\left\langle\sigma^{-1}\right\rangle^{-1} \mathbf{I}$ translates into the elementary bound $\boldsymbol{\sigma}^{D} \geq\left\langle\sigma^{-1}\right\rangle^{-1} \mathbf{I}$ of Nemat-Nasser and Hori, obtained before. Additionally, in our two-phase composite, the effective conductivity $\boldsymbol{\sigma}^{*}$ satisfies the Lurie-Cherkaev-MuratTartar bound (Lurie and Cherkaev 1982, 1984; Murat and Tartar 1985, Tartar 1985)

$$
f_{1} \operatorname{Tr}\left[\left(\boldsymbol{\sigma}^{*}-\sigma_{2} \mathbf{I}\right)^{-1}\right] \leq d /\left(\sigma_{1}-\sigma_{2}\right)+f_{2} / \sigma_{2},
$$

[which are a generalization of the bounds of Hashin and Shtrikman (1962)] where $d=2,3$ is the dimensionality of the composite. Since $\boldsymbol{\sigma}^{D} \geq \boldsymbol{\sigma}^{*} \geq \sigma_{2} \mathbf{I}$ it follows that

$$
\left(\boldsymbol{\sigma}^{*}-\sigma_{2} \mathbf{I}\right)^{-1} \geq\left(\boldsymbol{\sigma}^{D}-\sigma_{2} \mathbf{I}\right)^{-1},
$$

and so (5.10) implies the new bound

$$
f_{1} \operatorname{Tr}\left[\left(\boldsymbol{\sigma}^{D}-\sigma_{2} \mathbf{I}\right)^{-1}\right] \leq d /\left(\sigma_{1}-\sigma_{2}\right)+f_{2} / \sigma_{2} .
$$


By multiplying this inequality by $\sigma_{2}^{2}$ and adding $d f_{1} \sigma_{2}$ to both sides we see that it can be rewritten in the equivalent form

$$
f_{1} \operatorname{Tr}\left[\left(\sigma_{2}^{-1} \mathbf{I}-\left(\boldsymbol{\sigma}^{D}\right)^{-1}\right)^{-1}\right] \leq d /\left(\sigma_{2}^{-1}-\sigma_{1}^{-1}\right)-(d-1) f_{2} \sigma_{2} .
$$

As $d^{2} / \operatorname{Tr}(\mathbf{A}) \leq \operatorname{Tr}\left(\mathbf{A}^{-1}\right)$ for any positive definite matrix $\mathbf{A}$ we also obtain the weaker bound

$$
\frac{1}{d} \operatorname{Tr}\left[\left(\boldsymbol{\sigma}^{D}\right)^{-1}\right] \leq \sigma_{2}^{-1}-\frac{f_{1} d}{d /\left(\sigma_{2}^{-1}-\sigma_{1}^{-1}\right)-(d-1) f_{2} \sigma_{2}},
$$

which is a particular case of the universal bounds first derived by Nemat-Nasser and Hori (1993, 1995), see equation (5.4.9) in their 1995 paper, obtained under the assumption that $\Omega$ is ellipsoidal or parallelpipedic. (which we see is not needed).

If one is interested in bounds on the volume fraction $f_{1}$ then (5.12) implies the upper bound

$$
f_{1} \leq \frac{1 / \sigma_{2}+d /\left(\sigma_{1}-\sigma_{2}\right)}{1 / \sigma_{2}+\operatorname{Tr}\left[\left(\boldsymbol{\sigma}^{D}-\sigma_{2} \mathbf{I}\right)^{-1}\right]}
$$

To obtain lower bounds on $f_{1}$, we consider the same periodic composite and apply the dual variational inequality

$$
\mathbf{j}_{0} \cdot\left(\boldsymbol{\sigma}_{n}^{*}\right)^{-1} \mathbf{j}_{0} \leq\left\langle\underline{\mathbf{j}}_{C} \cdot \sigma_{C}^{-1} \underline{\mathbf{j}}_{C}\right\rangle
$$

valid for any trial current field $\underline{\mathbf{j}}_{C}$ satisfying

$$
\nabla \cdot \underline{\mathbf{j}}_{C}=0, \quad \underline{\mathbf{j}}_{C} \text { periodic, } \quad\left\langle\underline{\mathbf{j}}_{C}\right\rangle=\mathbf{j}_{0} .
$$

Letting $\mathbf{j}(\mathbf{x})$ denote the current field within $\Omega$ when the special Neumann boundary conditions (2.7) are applied, we may take in the unit cell

$$
\begin{aligned}
\underline{\mathbf{j}}_{C}(\mathbf{x}) & =\mathbf{j}\left(\mathbf{x} / a_{i}+\mathbf{b}_{i}\right) \text { in } \Omega_{i}, \quad i=1,2, \ldots, N \\
& =\mathbf{j}_{0} \text { elsewhere outside } \cup_{i} \Omega_{i},
\end{aligned}
$$

and periodically extend it. Substituting this trial field in (5.16) gives the bound

$$
\left(\boldsymbol{\sigma}_{n}^{*}\right)^{-1} \leq p_{n} \sigma_{2}^{-1} \mathbf{I}+\left(\boldsymbol{\sigma}^{N}\right)^{-1},
$$

which in the limit $n \rightarrow \infty$ implies

$$
\boldsymbol{\sigma}^{*} \geq \boldsymbol{\sigma}^{N}
$$

which is essentially the bound of Huet (1990) applied to the assemblage of rescaled copies of $\Omega$ packed to fill all space.

Thus any bound "from above" on the effective conductivity $\boldsymbol{\sigma}_{n}^{*}$ of composites having a volume fraction $f_{1}^{\prime}$ immediately translates into a bound "from above" on $\left(p_{n} \sigma_{2}^{-1} \mathbf{I}+\right.$ $\left.\left(\boldsymbol{\sigma}^{N}\right)^{-1}\right)^{-1}$. Taking the limit $n \rightarrow \infty$ and assuming continuity of the bound at $f_{1}^{\prime}=f_{1}$ the "upper bound" on the effective tensor of composites having volume fraction $f_{1}$ must also be an upper bound on $\boldsymbol{\sigma}^{N}$. In particular, the other Murat-Tartar-Lurie-Cherkaev bound

$$
f_{2} \operatorname{Tr}\left[\left(\sigma_{1} \mathbf{I}-\boldsymbol{\sigma}^{*}\right)^{-1}\right] \leq d /\left(\sigma_{1}-\sigma_{2}\right)-f_{1} / \sigma_{1}
$$

implies

$$
f_{2} \operatorname{Tr}\left[\left(\sigma_{1} \mathbf{I}-\boldsymbol{\sigma}^{N}\right)^{-1}\right] \leq d /\left(\sigma_{1}-\sigma_{2}\right)-f_{1} / \sigma_{1}
$$


Again using the inequality $d^{2} / \operatorname{Tr}(\mathbf{A}) \leq \operatorname{Tr}\left(\mathbf{A}^{-1}\right)$ for $\mathbf{A}>0$, we obtain the weaker bound

$$
\frac{1}{d} \operatorname{Tr}\left(\boldsymbol{\sigma}^{N}\right) \leq \sigma_{1}-\frac{f_{2} d}{d /\left(\sigma_{1}-\sigma_{2}\right)-f_{1} / \sigma_{1}}
$$

which is a particular case of the universal bounds derived by Nemat-Nasser and Hori (1993, 1995), see equation (5.3.11) in their 1995 paper, obtained under the assumption that $\Omega$ is ellipsoidal or parallelpipedic (which we see is not needed).

From (5.22) we directly obtain the volume fraction bound

$$
f_{2} \leq \frac{d /\left(\sigma_{1}-\sigma_{2}\right)-1 / \sigma_{1}}{\left\{\operatorname{Tr}\left[\left(\sigma_{1} \mathbf{I}-\boldsymbol{\sigma}^{N}\right)^{-1}\right]\right\}-1 / \sigma_{1}},
$$

giving a lower bound on the volume fraction $f_{1}=1-f_{2}$.

In the asymptotic limit as the volume fraction goes to zero the volume fraction bounds (5.15) and (5.24) reduce to those of Capdeboscq and Vogelius (2003, 2004), as shown in the two dimensional case by Kang, Kim, and Milton (2011). The paper of Kang, Kim and Milton also tests the bounds numerically, and their (two-dimensional) results show the bound (5.15) is typically close to the actual volume fraction for a variety of inclusions of phase 1 in a matrix of phase 2. Similarly we can expect that the bound (5.24) will be typically close to the actual volume fraction for a variety of inclusions of phase 2 in a matrix of phase 1 .

\section{Coupled bounds in two-dimensions}

The tensors $\boldsymbol{\sigma}^{D}$ and $\boldsymbol{\sigma}^{N}$ obviously depend on $\sigma_{1}$ and $\sigma_{2}$, i.e. $\boldsymbol{\sigma}^{D}=\boldsymbol{\sigma}^{D}\left(\sigma_{1}, \sigma_{2}\right)$ and $\boldsymbol{\sigma}^{N}=\boldsymbol{\sigma}^{N}\left(\sigma_{1}, \sigma_{2}\right)$. Let us assume we have measurements of these tensors for an additional pair of conductivities $\left(k_{1}, k_{2}\right)$, (which could be obtained, say from thermal, magnetic permeability, or diffusivity measurements) and let $\mathbf{k}^{D}$ and $\mathbf{k}^{N}$ denote these tensors,

$$
\mathbf{k}^{D}=\boldsymbol{\sigma}^{D}\left(k_{1}, k_{2}\right), \quad \mathbf{k}^{N}=\boldsymbol{\sigma}^{N}\left(k_{1}, k_{2}\right) .
$$

We still let $\boldsymbol{\sigma}^{D}$ and $\boldsymbol{\sigma}^{N}$ denote the tensors associated with the first pair of conductivities $\left(\sigma_{1}, \sigma_{2}\right)$, with $\sigma_{1}>\sigma_{2}$. From (5.9) and (5.20) we have the inequalities

$$
\begin{gathered}
\sigma_{2} \mathbf{I} \leq \boldsymbol{\sigma}^{N} \leq \boldsymbol{\sigma}^{*} \leq \boldsymbol{\sigma}^{D} \leq \sigma_{1} \mathbf{I} \\
k^{-} \leq \mathbf{k}^{N} \leq \mathbf{k}^{*} \leq \mathbf{k}^{D} \leq k^{+} \mathbf{I},
\end{gathered}
$$

where $k^{-}=\min \left\{k_{1}, k_{2}\right\}$ and $k^{+}=\max \left\{k_{1}, k_{2}\right\}$ and $\mathbf{k}^{*}$ is the effective conductivity the composite considered in the previous section when $\sigma_{1}$ and $\sigma_{2}$ are replaced by $k_{1}$ and $k_{2}$. (It can easily be checked that these inequalities still hold if $k_{2}>k_{1}$.)

For two dimensional conductivity from duality (Keller 1964; Dykhne 1970) we know the functions $\boldsymbol{\sigma}^{D}=\boldsymbol{\sigma}^{D}\left(\sigma_{1}, \sigma_{2}\right)$ and $\boldsymbol{\sigma}^{N}=\boldsymbol{\sigma}^{N}\left(\sigma_{1}, \sigma_{2}\right)$ satisfy

$$
\begin{aligned}
& \boldsymbol{\sigma}^{D}\left(\sigma_{2}, \sigma_{1}\right)=\sigma_{1} \sigma_{2} \mathbf{R}_{\perp}^{T}\left[\boldsymbol{\sigma}^{N}\left(\sigma_{1}, \sigma_{2}\right)\right]^{-1} \mathbf{R}_{\perp}, \\
& \boldsymbol{\sigma}^{N}\left(\sigma_{2}, \sigma_{1}\right)=\sigma_{1} \sigma_{2} \mathbf{R}_{\perp}^{T}\left[\boldsymbol{\sigma}^{D}\left(\sigma_{1}, \sigma_{2}\right)\right]^{-1} \mathbf{R}_{\perp},
\end{aligned}
$$

where

$$
\mathbf{R}_{\perp}=\left(\begin{array}{cc}
0 & 1 \\
-1 & 0
\end{array}\right)
$$


is the matrix for a $90^{\circ}$ rotation. So if we know these tensors for the conductivity pair $\left(k_{1}, k_{2}\right)$, we also know them for the conductivity pair $\left(k_{2}, k_{1}\right)$. Hence, by making such an interchange if necessary, we may assume without loss of generality that $k_{1}>k_{2}$, i.e. that $k^{+}=k_{1}$ and $k^{-}=k_{2}$. Finally, by interchanging $k$ with $\sigma$ if necessary, we may assume without loss of generality that

$$
\sigma_{1} / \sigma_{2} \geq k_{1} / k_{2}>1
$$

Optimal bounds on all possible matrix pairs $\left(\boldsymbol{\sigma}^{*}, \mathbf{k}^{*}\right)$ for composites having a prescribed volume fraction $f_{1}$ of phase 1 have been derived by Cherkaev and Gibiansky (1992), and extended to an arbitrary number of effective conductivity function values by Clark and Milton (1933). However it seems difficult to extract bounds on $f_{1}$ from these optimal bounds. Instead we consider a polycrystal checkerboard with conductivities

$$
\boldsymbol{\sigma}(\mathbf{x})=\mathbf{R}^{T}(\mathbf{x}) \boldsymbol{\sigma}^{*} \mathbf{R}(\mathbf{x}), \quad \mathbf{k}(\mathbf{x})=\mathbf{R}^{T}(\mathbf{x}) \mathbf{k}^{*} \mathbf{R}(\mathbf{x}), \quad \text { with } \mathbf{R}^{T}(\mathbf{x}) \mathbf{R}(\mathbf{x})=\mathbf{I},
$$

in which the rotation field $\mathbf{R}(\mathbf{x})$ is $\mathbf{I}$ in the "white squares" and $\mathbf{R}_{\perp}$ in the "black squares". By a result of Dykhne (1970) this material has effective conductivities $\left(\sigma_{*} \mathbf{I}, k_{*} \mathbf{I}\right)$ where

$$
\sigma_{*}=\sqrt{\operatorname{det} \boldsymbol{\sigma}^{*}}, \quad k_{*}=\sqrt{\operatorname{det} \mathbf{k}^{*}} .
$$

Now we replace the "white squares" by the limiting composite considered in the previous section (with structure much smaller than the size of the squares) and we replace the "black squares" by the limiting composite considered in the previous section, rotated by $90^{\circ}$. The resulting material is an isotropic composite of phases 1 and 2 and so the pair $\left(\sigma_{*}, k_{*}\right)$ satisfies the bounds of Milton (1981b),

$$
u\left(k_{*}\right) \leq \sigma_{*} \leq v\left(k_{*}\right),
$$

which are attained when the composite is an assemblage of doubly coated disks, where

$$
\begin{aligned}
& v\left(k_{*}\right)=\sigma_{1}-\frac{2 f_{2} \sigma_{1}\left(\sigma_{1}^{2}-\sigma_{2}^{2}\right)}{\left(f_{2} \sigma_{1}+f_{1} \sigma_{2}+\sigma_{1}\right)\left(\sigma_{1}+\sigma_{2}\right)+\left(\sigma_{1}-\sigma_{2}\right)^{2} \alpha_{1}\left(k_{*}\right)}, \\
& u\left(k_{*}\right) \equiv \sigma_{2}+\frac{2 f_{1} \sigma_{2}\left(\sigma_{1}^{2}-\sigma_{2}^{2}\right)}{\left(f_{2} \sigma_{1}+f_{1} \sigma_{2}+\sigma_{2}\right)\left(\sigma_{1}+\sigma_{2}\right)+\left(\sigma_{1}-\sigma_{2}\right)^{2} \alpha_{2}\left(k_{*}\right)},
\end{aligned}
$$

and

$$
\begin{aligned}
& \alpha_{1}\left(k_{*}\right)=\frac{\left(k_{1}+k_{2}\right)\left[2 f_{2} k_{1}\left(k_{1}-k_{2}\right) /\left(k_{1}-k_{*}\right)-\left(f_{2} k_{1}+f_{1} k_{2}+k_{1}\right)\right]}{\left(k_{1}-k_{2}\right)^{2}}, \\
& \alpha_{2}\left(k_{*}\right)=\frac{\left(k_{1}+k_{2}\right)\left[2 f_{1} k_{2}\left(k_{1}-k_{2}\right) /\left(k_{*}-k_{2}\right)-\left(f_{2} k_{1}+f_{1} k_{2}+k_{2}\right)\right]}{\left(k_{1}-k_{2}\right)^{2}} .
\end{aligned}
$$

Now for any two symmetric matrices $\mathbf{A}$ and $\mathbf{B}$ with $\mathbf{A} \geq \mathbf{B}>0$ we have $\mathbf{B}^{-1 / 2} \mathbf{A} \mathbf{B}^{-1 / 2} \geq$ $\mathbf{I}$, and so $\operatorname{det}\left(\mathbf{B}^{-1 / 2} \mathbf{A B}^{-1 / 2}\right) \geq 1$ implying $\operatorname{det}(\mathbf{A})>\operatorname{det}(\mathbf{B})$. Thus (6.2) and (6.7) imply

$$
\sigma_{2} \leq \sigma_{N} \leq \sigma_{*} \leq \sigma_{D} \leq \sigma_{1}, \quad k_{2} \leq k_{N} \leq k_{*} \leq k_{D} \leq k_{1}
$$

where we define

$$
\sigma_{N}=\sqrt{\operatorname{det} \boldsymbol{\sigma}_{N}}, \quad \sigma_{D}=\sqrt{\operatorname{det} \boldsymbol{\sigma}_{D}}, \quad k_{N}=\sqrt{\operatorname{det} \mathbf{k}_{N}}, \quad k_{D}=\sqrt{\operatorname{det} \mathbf{k}_{D}} .
$$


The Hashin-Shtrikman bounds (Hashin and Shtrikman 1962; Hashin 1970),

$$
k_{1}-\frac{2 f_{2} k_{1}\left(k_{1}-k_{2}\right)}{f_{2} k_{1}+f_{1} k_{2}+k_{1}} \geq k_{*} \geq k_{2}+\frac{2 f_{1} k_{2}\left(k_{1}-k_{2}\right)}{f_{2} k_{1}+f_{1} k_{2}+k_{2}},
$$

imply that both $\alpha_{1}\left(k_{*}\right)$ and $\alpha_{2}\left(k_{*}\right)$ are non-negative. Hence the denominators in (6.9) are positive and so (6.8) implies

$$
\begin{gathered}
\left(\sigma_{1}-\sigma_{*}\right)\left[\left(f_{2} \sigma_{1}+f_{1} \sigma_{2}+\sigma_{1}\right)\left(\sigma_{1}+\sigma_{2}\right)+\left(\sigma_{1}-\sigma_{2}\right)^{2} \alpha_{1}\left(k_{*}\right)\right] \geq 2 f_{2} \sigma_{1}\left(\sigma_{1}^{2}-\sigma_{2}^{2}\right), \\
\left(\sigma_{*}-\sigma_{2}\right)\left[\left(f_{2} \sigma_{1}+f_{1} \sigma_{2}+\sigma_{2}\right)\left(\sigma_{1}+\sigma_{2}\right)+\left(\sigma_{1}-\sigma_{2}\right)^{2} \alpha_{2}\left(k_{*}\right)\right] \geq 2 f_{1} \sigma_{2}\left(\sigma_{1}^{2}-\sigma_{2}^{2}\right) .
\end{gathered}
$$

Since $\alpha_{1}\left(k_{D}\right) \geq \alpha_{1}\left(k_{*}\right)$ and $\alpha_{2}\left(k_{N}\right) \geq \alpha_{2}\left(k_{*}\right)$, we get using (6.11),

$$
\begin{aligned}
& \left(\sigma_{1}-\sigma_{N}\right)\left[\left(f_{2} \sigma_{1}+f_{1} \sigma_{2}+\sigma_{1}\right)\left(\sigma_{1}+\sigma_{2}\right)+\left(\sigma_{1}-\sigma_{2}\right)^{2} \alpha_{1}\left(k_{D}\right)\right] \geq 2 f_{2} \sigma_{1}\left(\sigma_{1}^{2}-\sigma_{2}^{2}\right), \\
& \left(\sigma_{D}-\sigma_{2}\right)\left[\left(f_{2} \sigma_{1}+f_{1} \sigma_{2}+\sigma_{2}\right)\left(\sigma_{1}+\sigma_{2}\right)+\left(\sigma_{1}-\sigma_{2}\right)^{2} \alpha_{2}\left(k_{N}\right)\right] \geq 2 f_{1} \sigma_{2}\left(\sigma_{1}^{2}-\sigma_{2}^{2}\right) .
\end{aligned}
$$

As $\alpha_{1}\left(k_{D}\right)$ and $\alpha_{2}\left(k_{N}\right)$ depend linearly on $f_{1}$ and $f_{2}=1-f_{1}$, the equations (6.15) readily yield bounds on the volume fraction. Eunjoo Kim has used an integral equation solver [as described by Kang, Kim, and Milton (2011)] to compare the bounds (6.15) with the bounds (5.15) and (5.24). Her results are presented in figures 3, 4, and 5. More numerical results testing the bounds (5.15) and (5.24) are in the paper by Kang, Kim, and Milton (2011).
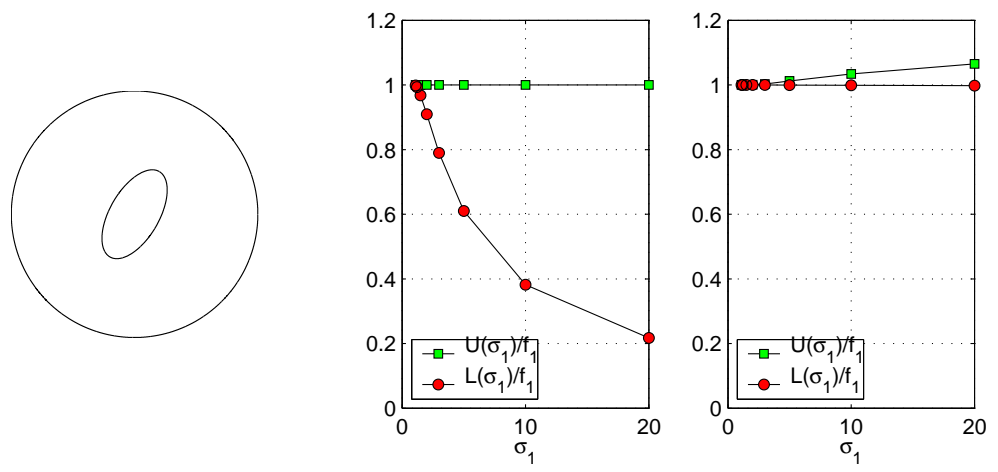

Figure 3: The first figure shows the circular body $\Omega$ containing an ellipse of phase 1 surrounded by phase 2. The second figure shows the results for the bounds (5.15) and (5.24) while the third figure shows the results for the bounds (6.15). The bounds are for increasing $\sigma_{1}$, with $\sigma_{2}=1$ and (for the third figure) the pairs $\left(\sigma_{1}, k_{1}\right)$ are taken as $(1.1,1.05),(1.2,1.1),(1.5,1.2),(2,1.5),(3,2),(5,3),(10,5)$ and $(20,10)$, with $\sigma_{2}=k_{2}=$ 1. Here $U\left(\sigma_{1}\right)$ and $L\left(\sigma_{1}\right)$ are the upper and lower bounds on the volume fraction, and the true volume fraction is $f_{1}=0.08$. Figure supplied courtesy of Eunjoo Kim.

\section{Coupled bounds in three-dimensions}

We can also derive coupled bounds in three dimensions. Let us assume the phases have been labeled so that

$$
\sigma_{1} k_{1} \geq \sigma_{2} k_{2}, \quad \text { i.e. } \sigma_{1} / \sigma_{2} \geq k_{2} / k_{1}
$$



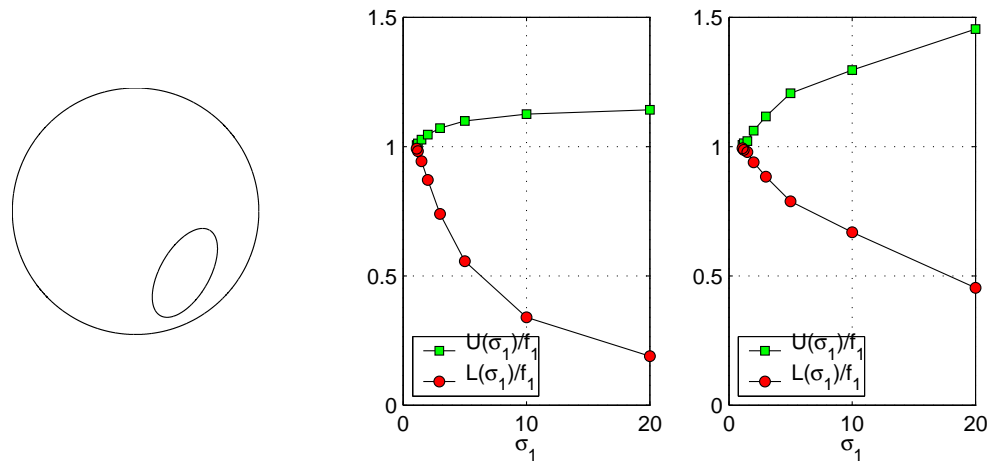

Figure 4: The same as for figure 3 but with the elliptical inclusion moved closer to the boundary of $\Omega$. Figure supplied courtesy of Eunjoo Kim.
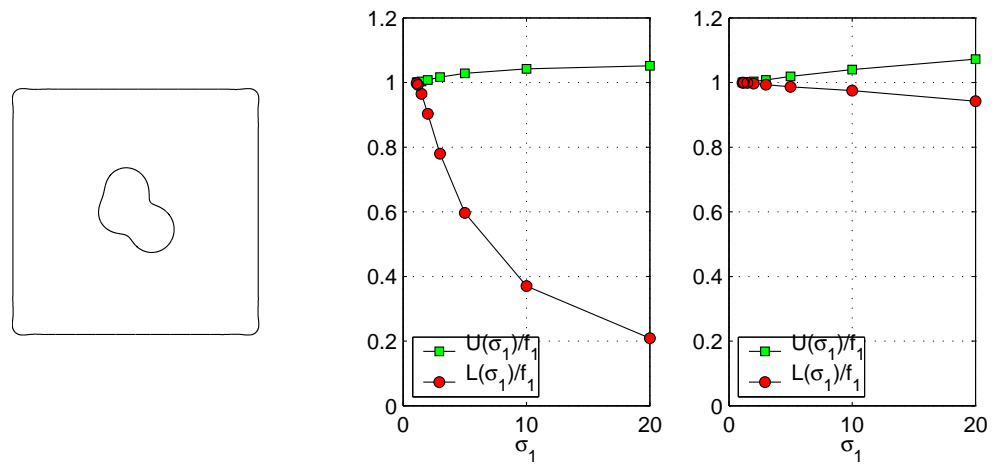

Figure 5: The same as for figure 3 but with a non-elliptical inclusion of phase 1 in a square region $\Omega$. The true volume fraction is $f_{1}=0.0673$. Figure supplied courtesy of Eunjoo Kim. 
and by interchanging $\sigma$ with $k$ if necessary let us assume

$$
\sigma_{1} / \sigma_{2} \geq k_{1} / k_{2}
$$

These two inequalities imply $\sigma_{1} / \sigma_{2}>1$ as before. We want to use the inequalities (6.2) to derive bounds on the volume fraction. As in the two-dimensional case the idea is to first construct an isotropic polycrystal, where the polycrystal has the conductivities (6.6) in which the rotation field $\mathbf{R}(\mathbf{x})$ is constant within grains which we take to be spheres. These spheres fill all space, and the crystal orientation varies randomly from sphere to sphere so that the composite has isotropic conductivities $\left(\sigma_{*} \mathbf{I}, k_{*} \mathbf{I}\right)$. We use the effective medium formula (Stroud 1975; Helsing and Helte 1991) which gives

$$
\sigma_{*}=g\left(\boldsymbol{\sigma}_{*}\right), \quad k_{*}=g\left(\mathbf{k}_{*}\right),
$$

where for any positive definite symmetric $3 \times 3$ matrix $\mathbf{A}, g=g(\mathbf{A})$ is taken to be the unique positive root of

$$
\frac{\lambda_{1}-g}{\lambda_{1}+2 g}+\frac{\lambda_{2}-g}{\lambda_{2}+2 g}+\frac{\lambda_{3}-g}{\lambda_{3}+2 g}=0
$$

in which $\lambda_{1}, \lambda_{2}$, and $\lambda_{3}$ are the eigenvalues of $\mathbf{A}$. This effective medium formula is realizable (Milton 1985; Avellaneda 1987) in the sense that it corresponds to a limiting composite of spherical grains with hierarchical structure (where any pair of grains of comparable size are well separated from each other, relative to their diameter). Note that the left hand side of side of (17.4) increases if any of the eigenvalues $\lambda_{i}$ increase, and decreases if $g$ increases. So $g(\mathbf{A})$ must increase if any or all of the eigenvalues of $\mathbf{A}$ increase. It follows that $g(\mathbf{B}) \geq g(\mathbf{A})$ if $\mathbf{B} \geq \mathbf{A}>0$. Hence the inequalities (6.2) imply

$$
\sigma_{2} \leq \sigma_{N} \leq \sigma_{*} \leq \sigma_{D} \leq \sigma_{1}, \quad k^{-} \leq k_{N} \leq k_{*} \leq k_{D} \leq k^{+}
$$

where now

$$
\sigma_{N}=g\left(\boldsymbol{\sigma}_{N}\right), \quad \sigma_{D}=g\left(\boldsymbol{\sigma}_{D}\right), \quad k_{N}=g\left(\mathbf{k}_{N}\right), \quad k_{D}=g\left(\mathbf{k}_{D}\right) .
$$

We next replace the material in each sphere by the appropriately oriented limiting composite considered in the previous section (with structure much smaller than the sphere diameter) to obtain a two-phase isotropic composite with $\left(\sigma_{*}, k_{*}\right)$ as its conductivities. Thus $\sigma_{*}$ must satisfy the upper bound of Bergman (1976 1978)

$$
\sigma_{*} \leq f_{1} \sigma_{1}+f_{2} \sigma_{2}-\frac{f_{1} f_{2}\left(\sigma_{1}-\sigma_{2}\right)^{2}}{3 \sigma_{2}+\left(\sigma_{1}-\sigma_{2}\right) \gamma\left(k_{*}\right)}
$$

where

$$
\gamma\left(k_{*}\right)=\frac{f_{1} f_{2}\left(k_{1}-k_{2}\right)}{f_{1} k_{1}+f_{2} k_{2}-k_{*}}-\frac{3 k_{2}}{k_{1}-k_{2}}
$$

and the lower bound

$$
\sigma_{*} \geq \sigma_{2}+\frac{3 f_{1} \sigma_{2}\left(\sigma_{1}-\sigma_{2}\right)\left(\sigma_{2}+2 \sigma_{1}\right)}{\left(f_{2} \sigma_{1}+f_{1} \sigma_{2}+2 \sigma_{2}\right)\left(\sigma_{2}+2 \sigma_{1}\right)+\left(\sigma_{1}-\sigma_{2}\right)^{2} \beta\left(k_{*}\right)},
$$


where

$$
\beta\left(k_{*}\right)=\frac{\left(k_{2}+2 k_{1}\right)\left[3 f_{1} k_{2}\left(k_{1}-k_{2}\right) /\left(k_{*}-k_{2}\right)-\left(f_{2} k_{1}+f_{1} k_{2}+2 k_{2}\right)\right]}{\left(k_{1}-k_{2}\right)^{2}} .
$$

This lower bound was first conjectured by Milton (1981b). A proof was proposed by Avellaneda, Cherkaev, Lurie, and Milton (1988) which was corrected by Nesi (1991) and Zhikov (1991).

The lower bound (7.9) is sharp, being attained for two-phase assemblages of doubly coated spheres (Milton 1981b). The upper bound (7.7) is attained at 5 values of $\gamma\left(k_{*}\right)$ namely when $\gamma\left(k_{*}\right)=f_{2}, 3 f_{2} / 2,3 f_{2}, 3-3 f_{1} / 2$, and $3-f_{1}$ (Milton 1981a).

The Hashin-Shtrikman bound (Hashin and Shtrikman 1962),

$$
\left(k_{*}-k_{2}\right) /\left(k_{1}-k_{2}\right) \geq 3 f_{1} k_{2} /\left(f_{2} k_{1}+f_{1} k_{2}+2 k_{2}\right),
$$

implies that $\beta\left(k_{*}\right)$ is non-negative. Hence the denominator in (7.9) is positive and so the inequality implies

$$
\left(\sigma_{*}-\sigma_{2}\right)\left[\left(f_{2} \sigma_{1}+f_{1} \sigma_{2}+2 \sigma_{2}\right)\left(\sigma_{2}+2 \sigma_{1}\right)+\left(\sigma_{1}-\sigma_{2}\right)^{2} \beta\left(k_{*}\right)\right] \geq 3 f_{1} \sigma_{2}\left(\sigma_{1}-\sigma_{2}\right)\left(\sigma_{2}+2 \sigma_{1}\right) .
$$

The Hashin-Shtrikman bounds can also be rewritten in the form

$$
f_{2} k_{1}+f_{1} k_{2}+2 k^{-} \leq \frac{f_{1} f_{2}\left(k_{1}-k_{2}\right)^{2}}{f_{1} k_{1}+f_{2} k_{2}-k_{*}} \leq f_{2} k_{1}+f_{1} k_{2}+2 k^{+} .
$$

These inequalities imply $\gamma\left(k_{*}\right)$ lies between $f_{2}$ and $3-f_{1}$. Hence the denominator in (17.7) is positive and the inequality can be rewritten as

$$
\left(f_{1} \sigma_{1}+f_{2} \sigma_{2}-\sigma_{*}\right)\left(3 \sigma_{2}+\left(\sigma_{1}-\sigma_{2}\right) \gamma\left(k_{*}\right)\right) \geq f_{1} f_{2}\left(\sigma_{1}-\sigma_{2}\right)^{2} .
$$

When $k_{1} \geq k_{2}$ (17.5) implies $\beta\left(k_{N}\right) \geq \beta\left(k_{*}\right)$ and $\gamma\left(k_{D}\right) \geq \gamma\left(k_{*}\right)$, and hence

$$
\begin{aligned}
\left(\sigma_{D}-\sigma_{2}\right)\left[\left(f_{2} \sigma_{1}+f_{1} \sigma_{2}+2 \sigma_{2}\right)\left(\sigma_{2}+2 \sigma_{1}\right)+\left(\sigma_{1}-\sigma_{2}\right)^{2} \beta\left(k_{N}\right)\right] & \geq 3 f_{1} \sigma_{2}\left(\sigma_{1}-\sigma_{2}\right)\left(\sigma_{2}+2 \sigma_{1}\right), \\
\left(f_{1} \sigma_{1}+f_{2} \sigma_{2}-\sigma_{N}\right)\left(3 \sigma_{2}+\left(\sigma_{1}-\sigma_{2}\right) \gamma\left(k_{D}\right)\right) & \geq f_{1} f_{2}\left(\sigma_{1}-\sigma_{2}\right)^{2} .
\end{aligned}
$$

On the other hand when $k_{1} \leq k_{2}$ then (7.5) implies $\beta\left(k_{D}\right) \geq \beta\left(k_{*}\right)$ and $\gamma\left(k_{N}\right) \geq \gamma\left(k_{*}\right)$, and hence

$$
\begin{aligned}
\left(\sigma_{D}-\sigma_{2}\right)\left[\left(f_{2} \sigma_{1}+f_{1} \sigma_{2}+2 \sigma_{2}\right)\left(\sigma_{2}+2 \sigma_{1}\right)+\left(\sigma_{1}-\sigma_{2}\right)^{2} \beta\left(k_{D}\right)\right] & \geq 3 f_{1} \sigma_{2}\left(\sigma_{1}-\sigma_{2}\right)\left(\sigma_{2}+2 \sigma_{1}\right), \\
\left(f_{1} \sigma_{1}+f_{2} \sigma_{2}-\sigma_{N}\right)\left(3 \sigma_{2}+\left(\sigma_{1}-\sigma_{2}\right) \gamma\left(k_{N}\right)\right) & \geq f_{1} f_{2}\left(\sigma_{1}-\sigma_{2}\right)^{2} .
\end{aligned}
$$

Since $\beta\left(k_{N}\right)$ and $\beta\left(k_{D}\right)$ depend linearly on the volume fractions $f_{1}$ and $f_{2}=1-f_{1}$, the first inequalities in (7.15) and (7.16) also depend linearly on the volume fraction and easily yield bounds on the volume fraction. On the other hand, finding bounds on the volume fraction from the second inequalities in (7.15) and (7.16), involves solving a cubic equation in $f_{1}$. So instead of analytically computing the roots of this cubic it is probably better to numerically search for the range of values of $f_{1}$ where the second inequalities in (7.15) and (7.16) are satisfied. 


\section{Bounds for elasticity}

Let us consider solutions to the linear elasticity equations

$$
\boldsymbol{\tau}(\mathbf{x})=\mathcal{C}(\mathbf{x}) \boldsymbol{\epsilon}(\mathbf{x}), \quad \nabla \cdot \boldsymbol{\theta}=0, \quad \boldsymbol{\epsilon}=\left(\nabla \mathbf{u}+(\nabla \mathbf{u})^{T}\right) / 2,
$$

within $\Omega$, where $\mathbf{u}(\mathbf{x}), \boldsymbol{\epsilon}(\mathbf{x})$ and $\boldsymbol{\tau}(\mathbf{x})$, are the displacement field, strain field, and stress field, and $\mathcal{C}(\mathbf{x})$ is the fourth order elasticity tensor field

$$
\mathcal{C}(\mathbf{x})=\chi(\mathbf{x}) \mathcal{C}^{1}+(1-\chi(\mathbf{x})) \mathcal{C}^{2}
$$

in which $\mathcal{C}^{1}$ and $\mathcal{C}^{2}$ are the elasticity tensors of the phases, assumed to be isotropic with elements,

$$
\mathcal{C}_{i j k \ell}^{h}=\mu_{h}\left(\delta_{i k} \delta_{j \ell}+\delta_{i \ell} \delta_{j k}\right)+\left(\kappa_{h}-2 \mu_{h} / d\right) \delta_{i j} \delta_{k \ell}, \quad h=1,2,
$$

in which $d=2$ or 3 is the dimensionality, and $\mu_{1}, \mu_{2}$ and $\kappa_{1}, \kappa_{2}$ are the shear and bulk moduli of the two phases. From boundary information on the displacement $\mathbf{u}_{0}(\mathbf{x})=\mathbf{u}(\mathbf{x})$ and traction $\mathbf{f}(\mathbf{x})=\boldsymbol{\tau}(\mathbf{x}) \cdot \mathbf{n}$ we can immediately determine, using integration by parts, volume averages such as

$$
\begin{aligned}
\langle\boldsymbol{\epsilon}: \boldsymbol{\tau}\rangle & =\frac{1}{|\Omega|} \int_{\partial \Omega} \mathbf{u} \cdot \mathbf{f} \\
\langle\boldsymbol{\epsilon}\rangle & =\frac{1}{|\Omega|} \int_{\partial \Omega}\left(\mathbf{n u}^{T}+\mathbf{u n}^{T}\right) / 2 \\
\langle\boldsymbol{\tau}\rangle & =\frac{1}{|\Omega|} \int_{\partial \Omega} \mathbf{x f}^{T}
\end{aligned}
$$

in which ": " denotes a contraction of two indices.

There are two natural sets of boundary conditions. For any symmetric matrix $\boldsymbol{\epsilon}_{0}$ we could prescribe the special Dirichlet boundary conditions

$$
\mathbf{u}(\mathbf{x})=\boldsymbol{\epsilon}_{0} \mathbf{x}, \quad \text { for } \mathbf{x} \in \partial \Omega
$$

and measure $\boldsymbol{\tau}_{0}=\langle\boldsymbol{\tau}\rangle$. Here, according to (18.4), $\boldsymbol{\epsilon}_{0}$ equals $\langle\boldsymbol{\epsilon}\rangle$. Since $\boldsymbol{\tau}_{0}$ is linearly related to $\boldsymbol{\epsilon}_{0}$ we can write

$$
\boldsymbol{\tau}_{0}=\mathcal{C}^{D} \epsilon_{0}
$$

which defines the elasticity tensor $\boldsymbol{\sigma}^{D}$ ( $D$ for Dirichlet). Alternatively for any symmetric matrix $\boldsymbol{\tau}_{0}$ we could prescribe the special Neumann boundary conditions

$$
\boldsymbol{\tau}(\mathbf{x}) \cdot \mathbf{n}=\boldsymbol{\tau}_{0} \cdot \mathbf{n}, \quad \text { for } \mathbf{x} \in \partial \Omega,
$$

and measure $\boldsymbol{\epsilon}_{0}=\langle\boldsymbol{\epsilon}\rangle$. Here, according to (8.4), $\boldsymbol{\tau}_{0}$ equals $\langle\boldsymbol{\tau}\rangle$. Since $\boldsymbol{\epsilon}_{0}$ is linearly related to $\boldsymbol{\tau}_{0}$ we can write

$$
\boldsymbol{\epsilon}_{0}=\left(\mathcal{C}^{N}\right)^{-1} \boldsymbol{\tau}_{0}
$$

which defines the elasticity tensor $\boldsymbol{\sigma}^{N}$ ( $D$ for Dirichlet). It is easy to check that $\mathcal{C}^{D}$ and $\mathcal{C}^{N}$ satisfy all the usual symmetries of elasticity tensors.

Directly analogous to (3.13) we have the bounds

$$
\left\langle\mathcal{C}^{-1}\right\rangle^{-1} \leq \mathcal{C}^{D} \leq\langle\mathcal{C}\rangle, \quad\left\langle\mathcal{C}^{-1}\right\rangle^{-1} \leq \mathcal{C}^{N} \leq\langle\mathcal{C}\rangle
$$


of (Nemat-Nasser and Hori 1993), and directly analogous to (3.14) for any boundary condition (not just the special boundary conditions (8.5) and (8.7)) we have the bounds

$$
\langle\boldsymbol{\epsilon} \cdot \boldsymbol{\tau}\rangle \geq \boldsymbol{\epsilon}_{0} \cdot \boldsymbol{\sigma}^{N} \boldsymbol{\epsilon}_{0}, \quad\langle\boldsymbol{\epsilon} \cdot \boldsymbol{\tau}\rangle \geq \boldsymbol{\tau}_{0}\left(\boldsymbol{\sigma}^{D}\right)^{-1} \boldsymbol{\tau}_{0},
$$

where $\boldsymbol{\epsilon}_{0}=\langle\boldsymbol{\epsilon}\rangle$ and $\boldsymbol{\tau}_{0}=\langle\boldsymbol{\tau}\rangle$, due to Willis in 1989 private communication to NematNasser and Hori and presented by Nemat-Nasser and Hori (1993).

Also directly analogous to (5.9) and (5.201) we have the bounds

$$
\mathcal{C}^{N} \leq \mathcal{C}^{*} \leq \mathcal{C}^{D}
$$

where $\mathcal{C}^{*}$ is the effective elasticity tensor of any assemblage of rescaled copies of $\Omega$ packed to fill all space. These are essentially the bounds of Huet (1990) applied to this assemblage. Thus "lower bounds" on $\mathcal{C}^{*}$ directly give "lower bounds" on $\mathcal{C}^{D}$ and "upper bounds" on $\mathcal{C}^{*}$ directly give "upper bounds" on $\mathcal{C}^{N}$. In particular, in two dimensions lower and upper bounds on $\boldsymbol{\epsilon}_{0} \cdot \mathcal{C}^{*} \boldsymbol{\epsilon}_{0}$ have been obtained by Gibiansky and Cherkaev (1984) (for the equivalent plate equation) and also by Allaire and Kohn (1993a). Assuming that the phases have been labeled so that $\mu_{1} \geq \mu_{2}\left(\kappa_{1}-\kappa_{2}\right.$ could be either positive or negative) and letting $\epsilon_{1}$ and $\epsilon_{2}$ denote the two eigenvalues $\boldsymbol{\epsilon}_{0}$, the bounds imply

$$
\begin{gathered}
\boldsymbol{\epsilon}_{0} \cdot \mathcal{C}^{D} \boldsymbol{\epsilon}_{0} \geq\left(\epsilon_{1}+\epsilon_{2}\right)^{2} /\left(f_{1} / \kappa_{1}+f_{2} / \kappa_{2}\right)+\left(\epsilon_{1}-\epsilon_{2}\right)^{2} /\left(f_{1} / \mu_{1}+f_{2} / \mu_{2}\right), \\
\text { if }\left|\kappa_{1}-\kappa_{2}\right|\left(f_{1} \mu_{2}+f_{2} \mu_{1}\right)\left|\epsilon_{1}+\epsilon_{2}\right| \leq\left|\mu_{1}-\mu_{2}\right|\left(f_{1} \kappa_{2}+f_{2} \kappa_{1}\right)\left|\epsilon_{1}-\epsilon_{2}\right| ; \\
\boldsymbol{\epsilon}_{0} \cdot \mathcal{C}^{D} \boldsymbol{\epsilon}_{0} \geq\left(\epsilon_{1}+\epsilon_{2}\right)^{2}\left(f_{1} \kappa_{1}+f_{2} \kappa_{2}\right)+\left(\epsilon_{1}-\epsilon_{2}\right)^{2}\left(f_{1} \mu_{1}+f_{2} \mu_{2}\right) \\
-f_{1} f_{2} \frac{\left[\left|\kappa_{1}-\kappa_{2}\right|\left|\epsilon_{1}+\epsilon_{2}\right|+\left|\mu_{1}-\mu_{2}\right|\left|\epsilon_{1}-\epsilon_{2}\right|\right]^{2}}{f_{1}\left(\mu_{2}+\kappa_{2}\right)+f_{2}\left(\mu_{1}+\kappa_{1}\right)} \\
\quad \text { if }\left(\mu_{2}+f_{1} \kappa_{2}+f_{2} \kappa_{1}\right)\left|\epsilon_{1}-\epsilon_{2}\right| \geq f_{2}\left|\kappa_{1}-\kappa_{2}\right|\left|\epsilon_{1}+\epsilon_{2}\right| \\
\text { and }\left|\kappa_{1}-\kappa_{2}\right|\left(f_{1} \mu_{2}+f_{2} \mu_{1}\right)\left|\epsilon_{1}+\epsilon_{2}\right| \geq\left|\mu_{1}-\mu_{2}\right|\left(f_{1} \kappa_{2}+f_{2} \kappa_{1}\right)\left|\epsilon_{1}-\epsilon_{2}\right| ; \\
\boldsymbol{\epsilon}_{0} \cdot \mathcal{C}^{D} \boldsymbol{\epsilon}_{0} \geq \mu_{2}\left(\epsilon_{1}-\epsilon_{2}\right)^{2}+\frac{\kappa_{1} \kappa_{2}+\mu_{2}\left(f_{1} \kappa_{1}+f_{2} \kappa_{2}\right)}{\mu_{2}+f_{1} \kappa_{2}+f_{2} \kappa_{1}}\left(\epsilon_{1}+\epsilon_{2}\right)^{2} \\
\text { if }\left(\mu_{2}+f_{1} \kappa_{2}+f_{2} \kappa_{1}\right)\left|\epsilon_{1}-\epsilon_{2}\right| \leq f_{2}\left|\kappa_{1}-\kappa_{2}\right|\left|\epsilon_{1}+\epsilon_{2}\right| ;
\end{gathered}
$$

and

$$
\begin{array}{r}
\boldsymbol{\epsilon}_{0} \cdot \mathcal{C}^{N} \boldsymbol{\epsilon}_{0} \leq\left(\epsilon_{1}+\epsilon_{2}\right)^{2}\left(f_{1} \kappa_{1}+f_{2} \kappa_{2}\right)+\left(\epsilon_{1}-\epsilon_{2}\right)^{2}\left(f_{1} \mu_{1}+f_{2} \mu_{2}\right) \\
\quad-f_{1} f_{2} \frac{\left[\left|\kappa_{1}-\kappa_{2}\right|\left|\epsilon_{1}+\epsilon_{2}\right|-\left|\mu_{1}-\mu_{2}\right|\left|\epsilon_{1}-\epsilon_{2}\right|\right]^{2}}{f_{1}\left(\mu_{2}+\kappa_{2}\right)+f_{2}\left(\mu_{1}+\kappa_{1}\right)}, \\
\text { if } f_{1}\left|\kappa_{1}-\kappa_{2}\right|\left|\epsilon_{1}+\epsilon_{2}\right| \leq\left(\mu_{1}+f_{1} \kappa_{2}+f_{2} \kappa_{1}\right)\left|\epsilon_{1}-\epsilon_{2}\right| \\
\text { and } f_{+}\left|\mu_{1}-\mu_{2}\right|\left|\epsilon_{1}-\epsilon_{2}\right| \leq\left(\kappa_{+}+f_{1} \mu_{2}+f_{2} \mu_{1}\right)\left|\epsilon_{1}+\epsilon_{2}\right| ; \\
\boldsymbol{\epsilon}_{0} \cdot \mathcal{C}^{N} \boldsymbol{\epsilon}_{0} \leq \mu_{1}\left(\epsilon_{1}-\epsilon_{2}\right)^{2}+\frac{\kappa_{1} \kappa_{2}+\mu_{1}\left(f_{1} \kappa_{1}+f_{2} \kappa_{2}\right)}{\mu_{1}+f_{1} \kappa_{2}+f_{2} \kappa_{1}}\left(\epsilon_{1}+\epsilon_{2}\right)^{2}, \\
\text { if } \quad f_{1}\left|\kappa_{1}-\kappa_{2}\right|\left|\epsilon_{1}+\epsilon_{2}\right| \geq\left(\mu_{1}+f_{1} \kappa_{2}+f_{2} \kappa_{1}\right)\left|\epsilon_{1}-\epsilon_{2}\right| ; \\
\boldsymbol{\epsilon}_{0} \cdot \mathcal{C}^{N} \boldsymbol{\epsilon}_{0} \leq \kappa_{+}\left(\epsilon_{1}+\epsilon_{2}\right)^{2}+\frac{\mu_{1} \mu_{2}+\kappa_{+}\left(f_{1} \mu_{1}+f_{2} \mu_{2}\right)}{\kappa_{+}+f_{1} \mu_{2}+f_{2} \mu_{1}}\left(\epsilon_{1}-\epsilon_{2}\right)^{2}, \\
\text { if } f_{+}\left|\mu_{1}-\mu_{2}\right|\left|\epsilon_{1}-\epsilon_{2}\right| \geq\left(\kappa_{+}+f_{1} \mu_{2}+f_{2} \mu_{1}\right)\left|\epsilon_{1}+\epsilon_{2}\right|,
\end{array}
$$


where $\kappa_{+}$is the maximum of $\kappa_{1}$ and $\kappa_{2}$ and $f_{+}$is the volume fraction of the material corresponding to $\kappa_{+}$.

The corresponding three-dimensional bounds follow directly from (8.11) and the bounds of Allaire and Kohn (1993b), but are not so explicit. Assuming that the Lame moduli

$$
\lambda_{1}=\kappa_{1}-2 \mu_{1} / 3 \text { and } \lambda_{2}=\kappa_{2}-2 \mu_{2} / 3
$$

of both phases are positive, and that the bulk and shear moduli of the two phases are well-ordered with

$$
\kappa_{1}>\kappa_{2}>0 \text { and } \mu_{1}>\mu_{2}>0
$$

these bounds are

$$
\begin{aligned}
& \boldsymbol{\epsilon}_{0}: \mathcal{C}^{D} \boldsymbol{\epsilon}_{0} \geq \boldsymbol{\epsilon}_{0}: \mathcal{C}_{2} \boldsymbol{\epsilon}_{0}+f_{1} \max _{\boldsymbol{\eta}}\left[2 \boldsymbol{\epsilon}_{0}: \boldsymbol{\eta}-\boldsymbol{\eta}:\left(\mathcal{C}_{1}-\mathcal{C}_{2}\right)^{-1} \boldsymbol{\eta}-f_{2} g(\boldsymbol{\eta})\right], \\
& \boldsymbol{\epsilon}_{0}: \mathcal{C}^{N} \boldsymbol{\epsilon}_{0} \geq \boldsymbol{\epsilon}_{0}: \mathcal{C}_{1} \boldsymbol{\epsilon}_{0}+f_{2} \min _{\boldsymbol{\eta}}\left[2 \boldsymbol{\epsilon}_{0}: \boldsymbol{\eta}+\boldsymbol{\eta}:\left(\mathcal{C}_{1}-\mathcal{C}_{2}\right)^{-1} \boldsymbol{\eta}-f_{1} h(\boldsymbol{\eta})\right]
\end{aligned}
$$

where $g(\boldsymbol{\eta})$ and $h(\boldsymbol{\eta})$ are function of the eigenvalues $\eta_{1}, \eta_{2}$, and $\eta_{3}$ of the symmetric matrix $\boldsymbol{\eta}$. Assuming that these are labeled with

$$
\eta_{1} \leq \eta_{2} \leq \eta_{3}
$$

we have

$$
\begin{aligned}
& g(\boldsymbol{\eta})=\frac{\left(\eta_{1}-\eta_{3}\right)^{2}}{4 \mu_{2}}+\frac{\left(\eta_{1}+\eta_{3}\right)^{2}}{4\left(\lambda_{2}+\mu_{2}\right)} \text { if } \eta_{3} \geq \frac{\lambda_{2}+2 \mu_{2}}{2\left(\lambda_{2}+\mu_{2}\right)}\left(\eta_{1}+\eta_{3}\right) \geq \eta_{1}, \\
& g(\boldsymbol{\eta})=\frac{\eta_{1}^{2}}{\lambda_{2}+2 \mu_{2}} \text { if } \eta_{1}>\frac{\lambda_{2}+2 \mu_{2}}{2\left(\lambda_{2}+\mu_{2}\right)}\left(\eta_{1}+\eta_{3}\right), \\
& g(\boldsymbol{\eta})=\frac{\eta_{3}^{2}}{\lambda_{2}+2 \mu_{2}} \text { if } \eta_{3}<\frac{\lambda_{2}+2 \mu_{2}}{2\left(\lambda_{2}+\mu_{2}\right)}\left(\eta_{1}+\eta_{3}\right),
\end{aligned}
$$

and

$$
h(\boldsymbol{\eta})=\frac{1}{\lambda_{1}+2 \mu_{1}} \min \left\{\eta_{1}^{2}, \eta_{2}^{2}, \eta_{3}^{2}\right\} .
$$

The bounds (8.12), (8.13) and (8.16) can be used in an inverse way to bound the volume fraction $f_{1}=1-f_{2}$, for a single experiment when for special Dirichlet conditions $\boldsymbol{\epsilon}_{0}$ is prescribed and $\boldsymbol{\tau}_{0}\left(=\mathcal{C}^{D} \boldsymbol{\epsilon}_{0}\right)$ is measured, or when for special Neumann conditions $\boldsymbol{\tau}_{0}$ is prescribed and $\boldsymbol{\epsilon}_{0}\left(=\mathcal{C}^{N} \boldsymbol{\epsilon}_{0}\right)$ is measured. Allaire and Kohn (1993b) also derive bounds on the complementary energy and these imply

$\boldsymbol{\tau}_{0}:\left(\mathcal{C}^{N}\right)^{-1} \boldsymbol{\tau}_{0} \geq \boldsymbol{\tau}_{0}: \mathcal{C}_{1}^{-1} \boldsymbol{\tau}_{0}+f_{2} \max _{\boldsymbol{\zeta}}\left[2 \boldsymbol{\tau}_{0}: \boldsymbol{\zeta}-\boldsymbol{\zeta}:\left(\mathcal{C}_{2}^{-1}-\mathcal{C}_{1}^{-1}\right)^{-1} \boldsymbol{\zeta}-f_{1} \boldsymbol{\zeta}: \mathcal{C}_{1} \boldsymbol{\zeta}+f_{1} h\left(\mathcal{C}_{1} \boldsymbol{\zeta}\right)\right]$

and

$\boldsymbol{\tau}_{0}:\left(\mathcal{C}^{D}\right)^{-1} \boldsymbol{\tau}_{0} \leq \boldsymbol{\tau}_{0}: \mathcal{C}_{2}^{-1} \boldsymbol{\tau}_{0}+f_{1} \min _{\boldsymbol{\zeta}}\left[2 \boldsymbol{\tau}_{0}: \boldsymbol{\zeta}+\boldsymbol{\zeta}:\left(\mathcal{C}_{2}^{-1}-\mathcal{C}_{1}^{-1}\right)^{-1} \boldsymbol{\zeta}-f_{2} \boldsymbol{\zeta}: \mathcal{C}_{2} \boldsymbol{\zeta}+f_{2} g\left(\mathcal{C}_{2} \boldsymbol{\zeta}\right)\right]$

The bound in (8.20) is particularly useful when $\boldsymbol{\tau}_{0}=-p \mathbf{I}$, corresponding to immersing the body $\Omega$ in a fluid with pressure $p$. Then from measurements of the resulting volume 
change of the body one can determine $\boldsymbol{\tau}_{0}:\left(\boldsymbol{C}^{N}\right)^{-1} \boldsymbol{\tau}_{0}=-p \operatorname{Tr} \boldsymbol{\epsilon}_{0}$. Let us assume $\lambda_{1}>0$ and set $\boldsymbol{\zeta}=\alpha \mathbf{I}+\mathbf{A}$, with $\mathbf{A}$ being a trace free matrix with eigenvalues $a_{1}, a_{2}$ and $a_{3}$. Then we have $\boldsymbol{\eta}=\mathcal{C}_{1} \boldsymbol{\zeta}=2 \mu_{1}(k \mathbf{I}+\mathbf{A})$ where $k=\alpha\left[1+3 \lambda_{1} /\left(2 \mu_{1}\right)\right]$. Substitution gives

$$
\begin{aligned}
& {\left[\boldsymbol{\zeta}: \mathcal{C}_{1} \boldsymbol{\zeta}-h\left(\mathcal{C}_{1} \boldsymbol{\zeta}\right)\right]-\alpha^{2}\left[\mathbf{I}: \mathcal{C}_{1} \mathbf{I}-h\left(\mathcal{C}_{1} \mathbf{I}\right)\right]} \\
& \quad=2 \mu_{1}\left[a_{1}^{2}+a_{2}^{2}+a_{3}^{2}-\frac{2 \mu_{1}}{\lambda_{1}+2 \mu_{1}} \min \left\{\left(k+a_{1}\right)^{2}-k^{2},\left(k+a_{2}\right)^{2}-k^{2},\left(k+a_{3}\right)^{2}-k^{2}\right\}\right] \\
& \quad \geq 2 \mu_{1}\left[a_{1}^{2}+a_{2}^{2}+a_{3}^{2}-\min \left\{2 a_{1} k+a_{1}^{2}, 2 a_{2} k+a_{2}^{2}, 2 a_{3} k+a_{3}^{2}\right\}\right],
\end{aligned}
$$

which is surely positive since $\min \left\{2 a_{1} k+a_{1}^{2}, 2 a_{2} k+a_{2}^{2}, 2 a_{3} k+a_{3}^{2}\right\} \leq a_{j}^{2}$ where $j$ is such that $k a_{j}$ is non-positive. (Note that $a_{1}, a_{2}$ and $a_{3}$ cannot all have the same sign since they sum to zero). Consequently when $\boldsymbol{\tau}_{0}=-p \mathbf{I}$ the maximum over $\boldsymbol{\zeta}$ in (8.20) is achieved when $\mathbf{A}=0$ and taking the maximum over $\alpha$ gives

$$
-p \operatorname{Tr} \boldsymbol{\epsilon}_{0} \geq p^{2}\left[\frac{1}{\kappa_{1}}+\frac{f_{2}}{\frac{\kappa_{1} \kappa_{2}}{\kappa_{1}-\kappa_{2}}+\frac{4 f_{1} \mu_{1} \kappa_{1}}{3 \kappa_{1}+4 \mu_{1}}}\right],
$$

or equivalently

$$
-p /\left(\operatorname{Tr} \boldsymbol{\epsilon}_{0}\right) \leq \kappa_{H S H}^{+} \equiv \kappa_{1}-\frac{f_{2}}{1 /\left(\kappa_{1}-\kappa_{2}\right)-f_{1} /\left(\kappa_{1}+4 \mu_{1} / 3\right)},
$$

where $\kappa_{H S H}^{+}$is the upper bulk modulus bound of Hashin and Shtrikman (1963) and Hill (1963). The inequality (8.23) can be rewritten as

$$
\frac{f_{2}}{-\left(\operatorname{Tr} \boldsymbol{\epsilon}_{0} / p\right)-1 / \kappa_{1}} \leq \frac{\kappa_{1} \kappa_{2}}{\kappa_{1}-\kappa_{2}}+\frac{4 f_{1} \mu_{1} \kappa_{1}}{3 \kappa_{1}+4 \mu_{1}}
$$

where we have used the fact that $-\left(\operatorname{Tr} \boldsymbol{\epsilon}_{0} / p\right)-1 / \kappa_{1}$ is positive $\left(\right.$ since $\boldsymbol{\tau}_{0}:\left(\boldsymbol{C}^{N}\right)^{-1} \boldsymbol{\tau}_{0} \geq$ $\boldsymbol{\tau}_{0}:\left(\mathcal{C}_{1}\right)^{-1} \boldsymbol{\tau}_{0}$ by $(\underline{8.9})$ ). This then yields the volume fraction bound

$$
f_{2} \leq \frac{\frac{\kappa_{1} \kappa_{2}}{\kappa_{1}-\kappa_{2}}+\frac{4 \mu_{1} \kappa_{1}}{3 \kappa_{1}+4 \mu_{1}}}{\frac{1}{-\left(\operatorname{Tr} \boldsymbol{\epsilon}_{0} / p\right)-1 / \kappa_{1}}+\frac{4 \mu_{1} \kappa_{1}}{3 \kappa_{1}+4 \mu_{1}}}
$$

which we expect to be closest to the actual volume fraction when phase 2 (the softer phase) is the inclusion phase. Thus the bound may be particularly effective for estimating the volume of cavities in a body. Note that if some granules of phase 1 lie within these cavities, then such granules will not contribute to this volume fraction estimate, but will contribute to the overall weight. If the weight of the body has been measured (and the density of phase 1 is known) this provides a way of estimating the volume of granules of phase 1 which lie within the cavities.

When multiple experiments have been done, and the full tensor $\mathcal{C}^{D}$ or $\mathcal{C}^{N}$ has been determined, then the "trace bounds" of Zhikov(1988, 1991) and Milton and Kohn (1988) can be used. (These generalize the well known Hashin-Shtrikman (1963) bounds to anisotropic elastic composites.) Define the two traces

$$
\operatorname{Tr}_{h} \mathcal{A}=A_{i i j j} / d, \quad \operatorname{Tr}_{s} \mathcal{A}=A_{i j i j}-\left(A_{i i j j} / d\right)
$$


for any fourth order tensor $\mathcal{A}$ with elements $A_{i j k \ell}$ in spatial dimension $d$. Then, assuming the moduli of the two-phases are well ordered satisfying (8.15), their lower and upper "bulk modulus type bounds" imply, through (8.11), the universal bounds

$$
\begin{aligned}
& f_{1} \operatorname{Tr}_{h}\left[\left(\mathcal{C}^{D}-\mathcal{C}_{2}\right)^{-1}\right] \leq \frac{1}{d\left(\kappa_{1}-\kappa_{2}\right)}+\frac{f_{2}}{d \kappa_{2}+2(d-1) \mu_{2}} \\
& f_{2} \operatorname{Tr}_{h}\left[\left(\mathcal{C}_{1}-\mathcal{C}^{N}\right)^{-1}\right] \leq \frac{1}{d\left(\kappa_{1}-\kappa_{2}\right)}-\frac{f_{1}}{d \kappa_{1}+2(d-1) \mu_{1}}
\end{aligned}
$$

while their lower and upper "shear modulus type bounds," imply the universal bounds

$$
\begin{aligned}
& f_{1} \operatorname{Tr}_{s}\left[\left(\mathcal{C}^{D}-\mathcal{C}_{2}\right)^{-1}\right] \leq \frac{(d-1)(d+2)}{4\left(\mu_{1}-\mu_{2}\right)}+\frac{d(d-1)\left(\kappa_{2}+2 \mu_{2}\right) f_{2}}{2 \mu_{2}\left(d \kappa_{2}+2(d-1) \mu_{2}\right)} \\
& f_{2} \operatorname{Tr}_{s}\left[\left(\mathcal{C}_{1}-\mathcal{C}^{N}\right)^{-1}\right] \leq \frac{(d-1)(d+2)}{4\left(\mu_{1}-\mu_{2}\right)}-\frac{d(d-1)\left(\kappa_{1}+2 \mu_{1}\right) f_{1}}{2 \mu_{1}\left(d \kappa_{1}+2(d-1) \mu_{1}\right)}
\end{aligned}
$$

Since these inequalities depend linearly on $f_{1}=1-f_{2}$ they can easily be inverted to obtain bounds on $f_{1}$ given $\mathcal{C}^{D}$ or $\mathcal{C}^{N}$.

As noted by Milton and Kohn (1988) the lower and upper "bulk modulus type bounds" are tighter than those obtained by Kantor and Bergman (1984) and Francfort and Murat (1986), which imply

$$
\begin{array}{r}
\operatorname{Tr}_{h} \mathcal{C}^{N} \leq d \kappa_{1}-\frac{f_{2}}{\frac{1}{d\left(\kappa_{1}-\kappa_{2}\right)}-\frac{f_{1}}{d \kappa_{1}+2(d-1) \mu_{1}}}, \\
1 / \operatorname{Tr}_{h}\left[\left(\mathcal{C}^{D}\right)^{-1}\right] \geq d \kappa_{2}+\frac{f_{1}}{\frac{1}{d\left(\kappa_{1}-\kappa_{2}\right)}+\frac{f_{2}}{d \kappa_{2}+2(d-1) \mu_{2}}} .
\end{array}
$$

For bodies $\Omega$ of ellipsoidal or parallelopipedic shape the universal bounds (8.30) were obtained by Nemat-Nasser and Hori (1993, 1995): see the equations (4.3.9) and (4.4.8), with $I=1$, in their 1995 paper. Their other bounds, with $I=2$, which incorporate the "shear responses" of the tensors $\mathcal{C}^{N}$ and $\mathcal{C}^{D}$ are improved upon by the bounds (8.29) as can be seen using the inequality

$$
\operatorname{Tr}_{s} \mathcal{A}^{-1} \geq(d-1)^{2}(d+2)^{2} /\left(4 \operatorname{Tr}_{s} \mathcal{A}\right)
$$

which holds for any positive definite fourth-order tensor $\mathcal{A}$.

\section{Acknowledgements}

Eunjoo Kim is deeply thanked for generously providing figures 3, 4, and 5, and for doing the numerical simulations which generated them. Additionally the author is grateful to Hyeonbae Kang and Michael Vogelius for stimulating his interest in this problem, and for their comments on an initial draft of the manuscript. The author is most thankful for support from the Mathematical Sciences Research Institute and the Simons foundation, through an Eisenbud fellowship, and from National Science Foundation through grant DMS-0707978. 


\section{References}

Alessandrini, G., A. Morassi, and E. Rosset 2002. Detecting cavities by electrostatic boundary measurements. Inverse Problems 18:1333-1353.

Alessandrini, G. and E. Rosset 1998. The inverse conductivity problem with one measurement: bounds on the size of the unknown object. SIAM Journal on Applied Mathematics 58:1060-1071.

Alessandrini, G., E. Rosset, and J. K. Seo 2000. Optimal size estimates for the inverse conductivity problem with one measurement. Proceedings of the American Mathematical Society 128:53-64.

Allaire, G. 2002. Shape optimization by the homogenization method. Berlin / Heidelberg / London / etc.: Springer-Verlag. 456 pp.

Allaire, G. and R. V. Kohn 1993a. Explicit optimal bounds on the elastic energy of a two-phase composite in two space dimensions. Quarterly of Applied Mathematics 51(4):675-699.

Allaire, G. and R. V. Kohn 1993b. Optimal bounds on the effective behavior of a mixture of two well-ordered elastic materials. Quarterly of Applied Mathematics 51 (4):643-674.

Avellaneda, M. 1987. Iterated homogenization, differential effective medium theory, and applications. Communications on Pure and Applied Mathematics (New York) 40(5):527-554.

Avellaneda, M., A. V. Cherkaev, K. A. Lurie, and G. W. Milton 1988. On the effective conductivity of polycrystals and a three-dimensional phase-interchange inequality. Journal of Applied Physics 63(10):4989-5003.

Benveniste, Y. and G. W. Milton 2003. New exact results for the effective electric, elastic, piezoelectric and other properties of composite ellipsoid assemblages. Journal of the Mechanics and Physics of Solids 51(10):1773-1813.

Bergman, D. J. 1976. Variational bounds on some bulk properties of a two-phase composite material. Physical Review B (Solid State) 14:1531-1542.

Bergman, D. J. 1978. The dielectric constant of a composite material - A problem in classical physics. Physics Reports 43(9):377-407.

Berryman, J. G. and R. V. Kohn 1990. Variational constraints for electrical-impedance tomography. Physical Review Letters 65(3):325-328.

Capdeboscq, Y. and M. S. Vogelius 2003. Optimal asymptotic estimates for the volume of internal inhomogeneities in terms of multiple boundary measurements. Mathematical Modelling and Numerical Analysis = Modelisation mathématique et analyse numérique: $M^{2} A N$ 37:227-240.

Capdeboscq, Y. and M. S. Vogelius 2004. A review of some recent work on impedance imaging for inhomogeneities of low volume fraction. In C. Conca, R. Manásevich, G. Uhlmann, and M. S. Vogelius (eds.), Partial differential equations and inverse problems, pp. 69-87. Providence, RI: American Mathematical Society. ISBN 0821834487. 
Cherkaev, A. V. 2000. Variational Methods for Structural Optimization. Berlin / Heidelberg / London / etc.: Springer-Verlag. xxvi + 545 pp. ISBN 0-387-98462-3. LCCN QA1.A647 vol. 140.

Cherkaev, A. V. and L. V. Gibiansky 1992. The exact coupled bounds for effective tensors of electrical and magnetic properties of two-component two-dimensional composites. Proceedings of the Royal Society of Edinburgh. Section A, Mathematical and Physical Sciences 122(1-2):93-125.

Clark, K. E. and G. W. Milton 1933. Optimal bounds correlating electric, magnetic and thermal properties of two-phase, two-dimensional composites. Proceedings of the Royal Society of London. Series A, Mathematical and Physical Sciences 448 (1933):161-190.

Dykhne, A. M. 1970. Conductivity of a two-dimensional two-phase system. Zhurnal eksperimental'noi $i$ teoreticheskoi fiziki / Akademiia Nauk SSSR 59:110-115. English translation in Soviet Physics JETP 32:63-65 (1971).

Francfort, G. A. and F. Murat 1986. Homogenization and optimal bounds in linear elasticity. Archive for Rational Mechanics and Analysis 94(4):307-334.

Gibiansky, L. V. and A. V. Cherkaev 1984. Design of composite plates of extremal rigidity. Technical Report 914, Ioffe Physicotechnical Institute, Leningrad, USSR. 1-60 pp. English translation in Topics in the Mathematical Modelling of Composite Materials, pp. 95-137, ed. by A. Cherkaev and R. Kohn. ISBN 0-8176-3662-5.

Hashin, Z. 1970. Mechanics of composite materials. In F. W. Wendt, H. Liebowitz, and N. Perrone (eds.), Theory of Composite Materials, pp. 201-242. New York: Pergamon.

Hashin, Z. and S. Shtrikman 1962. A variational approach to the theory of the effective magnetic permeability of multiphase materials. Journal of Applied Physics 33:31253131 .

Hashin, Z. and S. Shtrikman 1963. A variational approach to the theory of the elastic behavior of multiphase materials. Journal of the Mechanics and Physics of Solids $11: 127-140$.

Helsing, J. and A. Helte 1991. Effective conductivity of aggregates of anisotropic grains. Journal of Applied Physics 69(6):3583-3588.

Hill, R. 1963. Elastic properties of reinforced solids: Some theoretical principles. Journal of the Mechanics and Physics of Solids 11:357-372.

Hori, M. and S. Nemat-Nasser 1995. Universal bounds for overall properties of linear and nonlinear heterogeneous solids. ASME Journal of Engineering Materials and Technology 117(4):412-432.

Hori, M. and S. Nemat-Nasser 1998. Universal bounds for effective piezoelectric moduli. Mechanics of Materials: An International Journal 30:1-19.

Huet, C. 1990. Application of variational concepts to size effects in elastic heterogeneous bodies. Journal of the Mechanics and Physics of Solids 38(6):813-841.

Ikehata, M. 1998. Size estimation of inclusion. Journal of Inverse and Ill-Posed Problems 6(2):127-140. 
Kang, H., E. Kim, and G. W. Milton 2011. Sharp bounds on the volume fractions of two materials in a two-dimensional body from electrical boundary measurements: the translation method. Calculus of Variations and Partial Differential Equations 28. Submitted.

Kang, H., J. K. Seo, and D. Sheen 1997. The inverse conductivity problem with one measurement: stability and estimation of size. SIAM Journal on Mathematical Analysis 28:1389-1405.

Kantor, Y. and D. J. Bergman 1984. Improved rigorous bounds on the effective elastic moduli of a composite material. Journal of the Mechanics and Physics of Solids 32:41-62.

Keller, J. B. 1964. A theorem on the conductivity of a composite medium. Journal of Mathematical Physics 5(4):548-549.

Lurie, K. A. and A. V. Cherkaev 1982. Accurate estimates of the conductivity of mixtures formed of two materials in a given proportion (two-dimensional problem). Doklady Akademii Nauk SSSR 264:1128-1130. English translation in Soviet Phys. Dokl. 27:461-462 (1982).

Lurie, K. A. and A. V. Cherkaev 1984. Exact estimates of conductivity of composites formed by two isotropically conducting media taken in prescribed proportion. Proceedings of the Royal Society of Edinburgh. Section A, Mathematical and Physical Sciences 99(1-2):71-87.

Milton, G. W. 1981a. Bounds on the complex permittivity of a two-component composite material. Journal of Applied Physics 52(8):5286-5293.

Milton, G. W. 1981b. Bounds on the transport and optical properties of a twocomponent composite material. Journal of Applied Physics 52(8):5294-5304.

Milton, G. W. 1985. The coherent potential approximation is a realizable effective medium scheme. Communications in Mathematical Physics 99(4):463-500.

Milton, G. W. 1990. On characterizing the set of possible effective tensors of composites: The variational method and the translation method. Communications on Pure and Applied Mathematics (New York) 43(1):63-125.

Milton, G. W. 2002. The Theory of Composites, pp. 295-298. Cambridge, United Kingdom: Cambridge University Press. Series editors: P.G.Ciarlet, A.Iserles, R.V.Kohn, and M.H.Wright. ISBN 0-521-78125-6. LCCN TA418.9.C6 M58 2001.

Milton, G. W. and R. V. Kohn 1988. Variational bounds on the effective moduli of anisotropic composites. Journal of the Mechanics and Physics of Solids 36(6):597629.

Murat, F. and L. Tartar 1985. Calcul des variations et homogénísation. (French) [Calculus of variation and homogenization]. In Les méthodes de l'homogénéisation: théorie et applications en physique, pp. 319-369. Paris: Eyrolles. English translation in Topics in the Mathematical Modelling of Composite Materials, pp. 139-173, ed. by A. Cherkaev and R. Kohn, ISBN 0-8176-3662-5. LCCN QC20.5 .M47 1985; TA418.9.C6 M473 1985. 
Nemat-Nasser, S. and M. Hori 1993. Micromechanics: Overall Properties of Heterogeneous Materials (First ed.). Amsterdam: North-Holland Publishing Co. xx +687 pp. ISBN 0-444-89881-6. LCCN TA418.9.I53 N46 1993.

Nesi, V. 1991. Multiphase interchange inequalities. Journal of Mathematical Physics $32(8): 2263-2275$.

Rauttum, U. Ё. 1983. Questions of the existence of a solution in problems of optimal control of leading coefficients of linear elliptic equations. Differentsial'nye Uravneniya 19(6):1040-1047. English translation in Differential Equations 19(6):775783 (1983).

Stroud, D. 1975. Generalized effective-medium approach to the conductivity of an inhomogeneous material. Physical Review B (Solid State) 12(8):3368-3373.

Tartar, L. 1979. Estimation de coefficients homogénéisés. (French) [Estimation of homogenization coefficients]. In R. Glowinski and J.-L. Lions (eds.), Computing Methods in Applied Sciences and Engineering: Third International Symposium, Versailles, France, December 5-9, 1977,, pp. 364-373. Berlin / Heidelberg / London / etc.: Springer-Verlag. English translation in Topics in the Mathematical Modelling of Composite Materials, pp. 9-20, ed. by A. Cherkaev and R. Kohn. ISBN 0-81763662-5. ISBN 0-387-09123-8.

Tartar, L. 1985. Estimations fines des coefficients homogénéisés. (French) [Fine estimations of homogenized coefficients]. In P. Krée (ed.), Ennio de Giorgi Colloquium: Papers Presented at a Colloquium Held at the H. Poincaré Institute in November 1983, pp. 168-187. London: Pitman Publishing Ltd. ISBN 0-273-08680-4. LCCN QA377.E56 1983.

Tartar, L. 1995. Remarks on the homogenization method in optimal design methods. In Proceedings of the International Conference: Homogenization and Applications to Material Sciences: Held in Nice, June 6-10, 1995, pp. 393-412. Tokyo: Gakkōtosho. ISBN 4-7625-0418-1.

Tartar, L. 2009. The General Theory of Homogenization: A Personalized Introduction. Berlin / Heidelberg / London / etc.: Springer-Verlag. ISBN 978-3-642-05194-4.

Zhikov, V. V. 1988. Estimates for the trace of an averaged tensor. Doklady Akademii Nauk SSSR 299(4):796-800. English translation in Soviet Math. Dokl. 37:456-459 (1988).

Zhikov, V. V. 1991. Estimates for the homogenized matrix and the homogenized tensor. Uspekhi Matematicheskikh Nauk = Russian Mathematical Surveys 46:49-109. English translation in Russ. Math. Surv. 46(3):65-136 (1991).

Zhikov, V. V., S. M. Kozlov, and O. A. Oleinik 1994. Homogenization of Differential Operators and Integral Functionals. Berlin / Heidelberg / London / etc.: Springer-Verlag. xi + 570 pp. ISBN 3-540-54809-2 (Berlin), 0-387-54809-2 (New York). LCCN QA377.Z45 1994. 

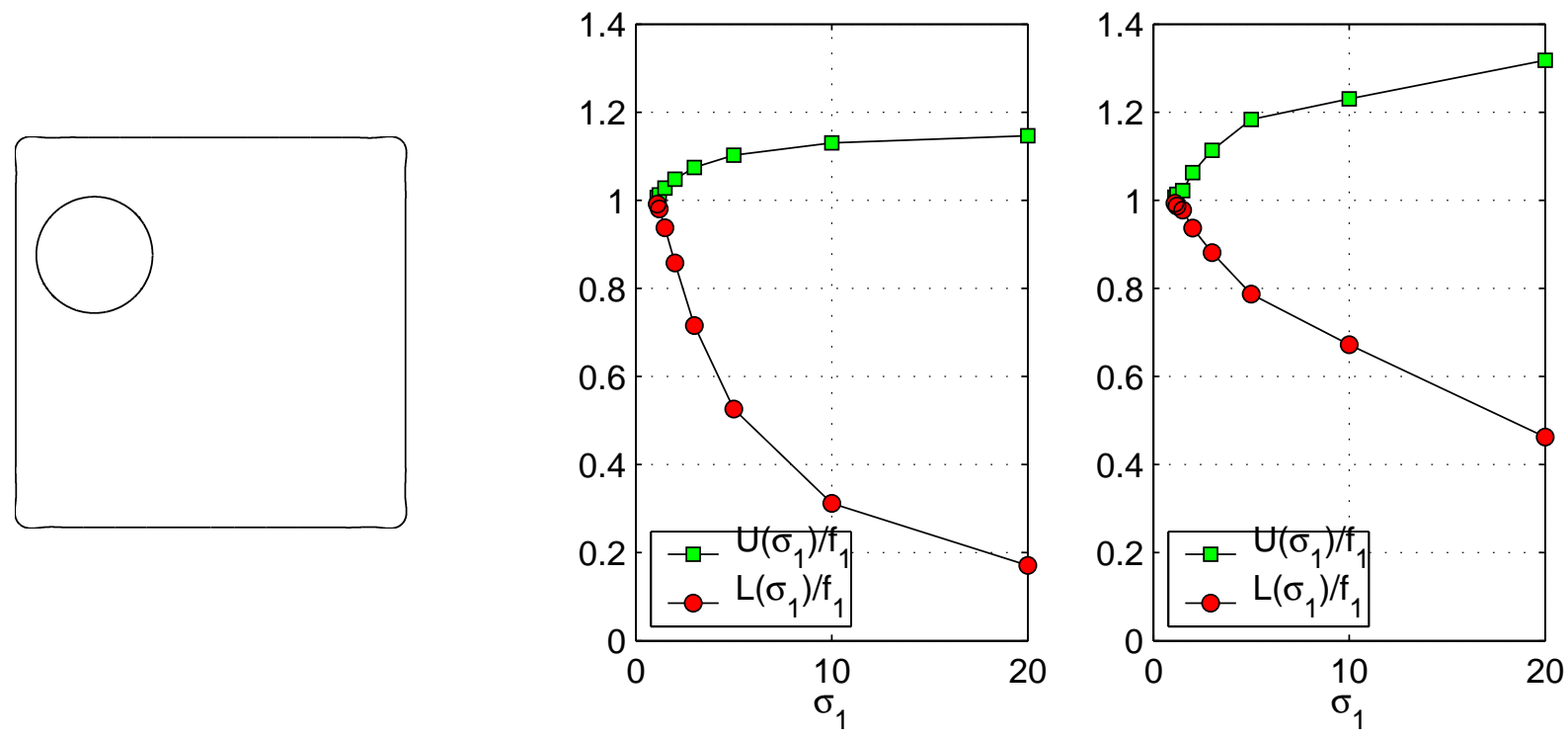

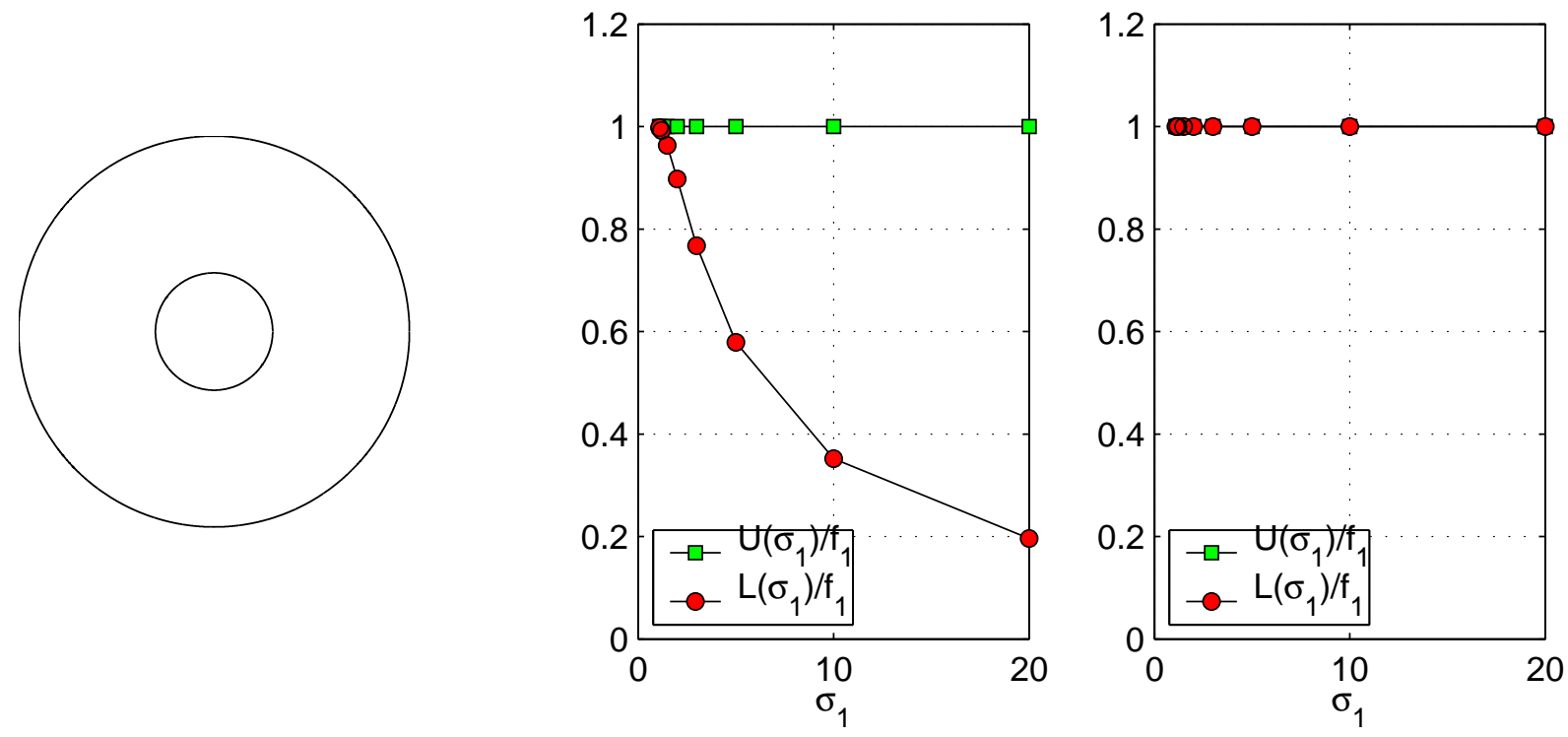

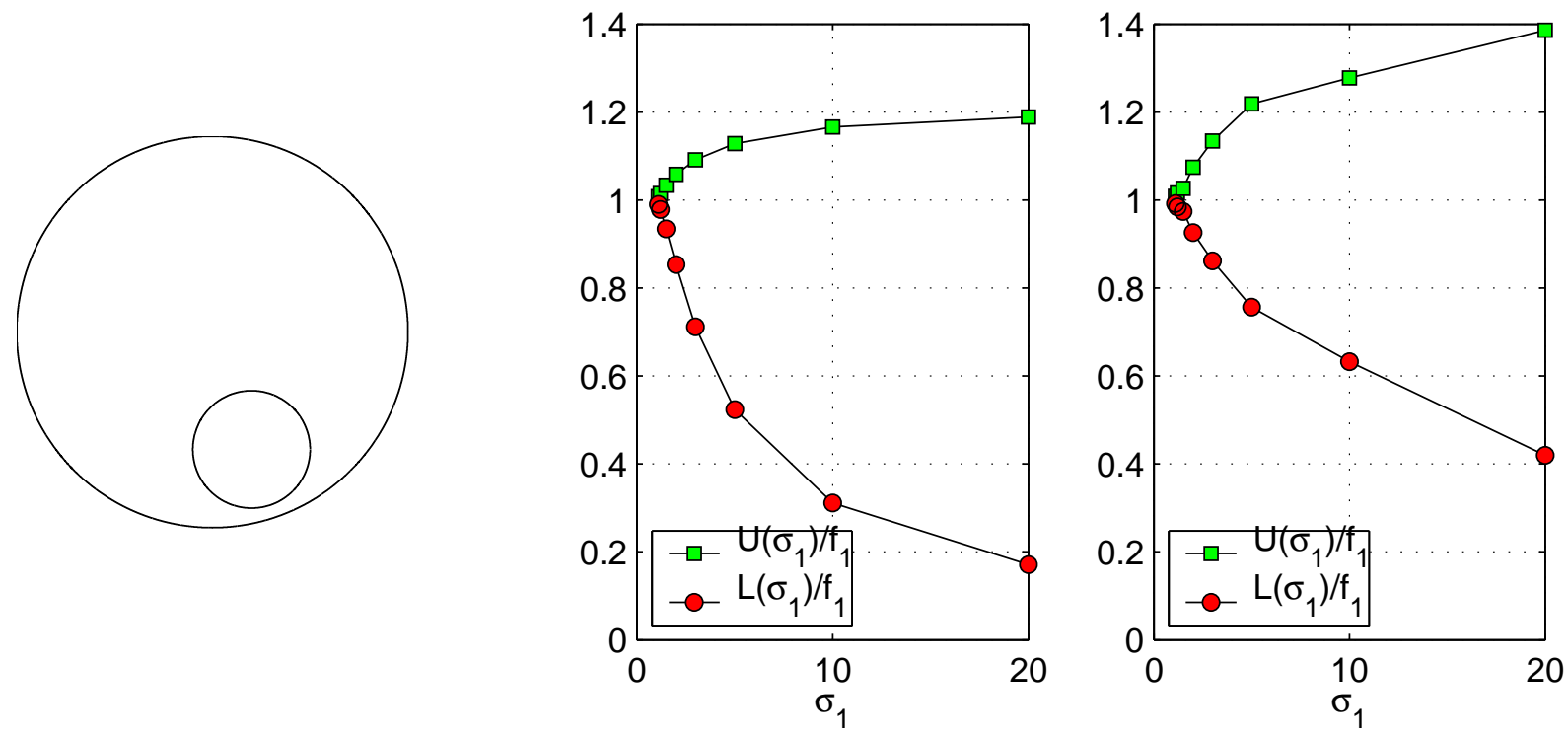